\title{
НЕКОТОРЫЕ ДОПОЛНЕНИЯ К НЕРАВЕНСТВАМ С. Б. СТЕЧКИНА ПО ПРЯМЫМ И ОБРАТНЫМ ТЕОРЕМАМ ТЕОРИИ ПРИБЛИЖЕНИЙ НЕПРЕРЫВНЫХ ПЕРИОДИЧЕСКИХ ФУНКЦИЙ
}

\author{
Н. А. Ильясов
}

\author{
Посвящается светлой памяти моего \\ учителя С. Б. Стечкина - удивителвного \\ в своей оригинальности и уникального \\ в своей индивидуальности Человека
}

\begin{abstract}
В статье приводятся некоторые дополнения и комментарии к неравенствам между элементами последовательности наилучших приближений $\left\{E_{n-1}(f)\right\}_{n=1}^{\infty}$ и модулями гладкости $k$-го порядка $\omega_{k}\left(f^{(r)} ; \delta\right)$, $\delta \in[0,+\infty)$, функции $f \in C^{r}(\mathbb{T})$, где $k \in \mathbb{N}, r \in \mathbb{Z}_{+}, f^{(0)} \equiv f, C^{0}(\mathbb{T}) \equiv C(\mathbb{T}), \mathbb{T}=(-\pi, \pi]$, полученным С.Б. Стечкиным при исследовании прямых и обратных теорем теории приближений $2 \pi$-периодических непрерывных функций и опубликованным в 1951 году. В работе, в частности, установлена справедливость следующих утверждений:
\end{abstract}

a) прямая теорема, или неравенство Джексона - Стечкина: $E_{n-1}(f) \leq C_{1}(k) \omega_{k}(f ; \pi / n), n \in \mathbb{N}$, допускает усиление вида $E_{n-1}(f) \leq \rho_{n}^{(k)}(f) \equiv n^{-k} \max \left\{\nu^{k} E_{\nu-1}(f): 1 \leq \nu \leq n\right\} \leq 2^{k} C_{1}(k) \omega_{k}(f ; \pi / n), n \in \mathbb{N}$, которое является точным в смысле порядка на классе всех функций $f \in C(\mathbb{T})$ с заданной мажорантой либо с заданным порядком убывания модуля гладкости $\omega_{k}(f ; \delta)$, а именно: для любых $k \in \mathbb{N}$ и $\omega \in \Omega_{k}(0, \pi]$ существует функция $f_{0}(\cdot ; \omega) \in C(\mathbb{T})$ (точнее, четная $f_{0}$ при нечетном $k$ и нечетная $f_{0}$ при четном $k$ ) такая, что $\omega_{k}\left(f_{0} ; \delta\right) \asymp C_{2}(k) \omega(\delta), \delta \in(0, \pi]$, при этом имеют место порядковые равенства $E_{n-1}\left(f_{0}\right) \asymp C_{3}(k) \rho_{n}^{(k)}\left(f_{0}\right) \asymp C_{4}(k) \omega_{k}\left(f_{0} ; \pi / n\right) \asymp C_{5}(k) \omega(\pi / n), n \in \mathbb{N}$, где $\Omega_{k}(0, \pi]-$ класс функций $\omega=\omega(\delta)$, определенных на $(0, \pi]$ и удовлетворяющих условиям: $0<\omega(\delta) \downarrow 0(\delta \downarrow 0)$ и $\delta^{-k} \omega(\delta) \downarrow(\delta \uparrow)$;

b) для справедливости обратной теоремы (без производных), или неравенства Салема - Стечкина: $\omega_{k}(f ; \pi / n) \leq C_{6}(k) n^{-k} \sum_{\nu=1}^{n} \nu^{k-1} E_{\nu-1}(f), n \in \mathbb{N}$, необходимо и достаточно выполнение неравенства Стечкина $\left\|T_{n}^{(k)}(f)\right\| \leq C_{7}(k) \sum_{\nu=1}^{n} \nu^{k-1} E_{\nu-1}(f), n \in \mathbb{N}$, где $T_{n}(f) \equiv T_{n}(f ; x)$ - тригонометрический полином наилучшего в $C(\mathbb{T})$ приближения функции $f:\left\|f-T_{n}(f)\right\|=E_{n}(f), n \in \mathbb{Z}_{+}$;

с) обратную теорему (с производными), или неравенство Валле-Пуссена - Стечкина: $\omega_{k}\left(f^{(r)} ; \pi / n\right) \leq$ $C_{8}(k, r)\left\{n^{-k} \sum_{\nu=1}^{n} \nu^{k+r-1} E_{\nu-1}(f)+\sum_{\nu=n+1}^{\infty} \nu^{r-1} E_{\nu-1}(f)\right\}, n \in \mathbb{N}$, а также предшествующее ему неравенство Стечкина $E_{n-1}\left(f^{(r)}\right) \leq C_{9}(r)\left\{n^{r} E_{n-1}(f)+\sum_{\nu=n+1}^{\infty} \nu^{r-1} E_{\nu-1}(f)\right\}, n \in \mathbb{N}$, при условии $E(f ; r) \equiv$ $\sum_{n=1}^{\infty} n^{r-1} E_{n-1}(f)<\infty$, гарантирующем в силу теоремы С. Н. Бернштейна, что $f \in C^{r}(\mathbb{T})$, где $r \in \mathbb{N}$, можно дополнить ключевыми неравенствами $\left\|f^{(r)}\right\| \leq C_{10}(r) E(f ; r),\left\|T_{n}^{(r)}(f)\right\| \leq C_{7}(r) \sum_{\nu=1}^{n} \nu^{r-1} E_{\nu-1}(f)$, $n \in \mathbb{N}$, при этом все сформулированные в этом пункте неравенства являются попарно равносильными, т. е. выполнение хотя бы одного из этих неравенств влечет выполнение любого другого неравенства и, следовательно, всех остальных.

Ключевые слова: наилучшее приближение, модуль гладкости, прямая теорема, обратная теорема, порядковое равенство, равносильные неравенства, точное в смысле порядка неравенство на классе.

N. A. Il'yasov. Some supplements to S. B. Stechkin's inequalities in direct and inverse theorems on the approximation of continuous periodic functions.

We give some supplements and comments to inequalities between elements of the sequence of best approximations $\left\{E_{n-1}(f)\right\}_{n=1}^{\infty}$ and the $k$ th-order moduli of smoothness $\omega_{k}\left(f^{(r)} ; \delta\right), \delta \in[0,+\infty)$, of a function $f \in C^{r}(\mathbb{T})$, where $k \in \mathbb{N}, r \in \mathbb{Z}_{+}, f^{(0)} \equiv f, C^{0}(\mathbb{T}) \equiv C(\mathbb{T})$, and $\mathbb{T}=(-\pi, \pi]$, which were published by S. B. Stechkin in 1951 in the study of direct and inverse theorems of approximation of $2 \pi$-periodic continuous functions. In particular, we prove the following results:

(a) the direct theorem or the Jackson-Stechkin inequality: $E_{n-1}(f) \leq C_{1}(k) \omega_{k}(f ; \pi / n), n \in \mathbb{N}$, can be strengthened as $E_{n-1}(f) \leq \rho_{n}^{(k)}(f) \equiv n^{-k} \max \left\{\nu^{k} E_{\nu-1}(f): 1 \leq \nu \leq n\right\} \leq 2^{k} C_{1}(k) \omega_{k}(f ; \pi / n), n \in \mathbb{N}$. This inequality is order-sharp on the class of all functions $f \in C(\mathbb{T})$ with a given majorant or with a given decrease order of the modulus of smoothness $\omega_{k}(f ; \delta)$; namely: for any $k \in \mathbb{N}$ and $\omega \in \Omega_{k}(0, \pi]$, there exists a function $f_{0}(\cdot ; \omega) \in C(\mathbb{T})\left(f_{0}\right.$ is even for odd $k$ and is odd for even $\left.k\right)$ such that $\omega_{k}\left(f_{0} ; \delta\right) \asymp C_{2}(k) \omega(\delta), \delta \in(0, \pi]$. Moreover, order equalities hold: $E_{n-1}\left(f_{0}\right) \asymp C_{3}(k) \rho_{n}^{(k)}\left(f_{0}\right) \asymp C_{4}(k) \omega_{k}\left(f_{0} ; \pi / n\right) \asymp C_{5}(k) \omega(\pi / n), n \in \mathbb{N}$, where 
$\Omega_{k}(0, \pi]$ is the class of functions $\omega=\omega(\delta)$ defined on $(0, \pi]$ and such that $0<\omega(\delta) \downarrow 0(\delta \downarrow 0)$ and $\delta^{-k} \omega(\delta) \downarrow$ $(\delta \uparrow)$

(b) a necessary and sufficient condition under which the inverse theorem (without the derivatives), or the Salem-Stechkin inequality $\omega_{k}(f ; \pi / n) \leq C_{6}(k) n^{-k} \sum_{\nu=1}^{n} \nu^{k-1} E_{\nu-1}(f), n \in \mathbb{N}$, holds is Stechkin's inequality $\left\|T_{n}^{(k)}(f)\right\| \leq C_{7}(k) \sum_{\nu=1}^{n} \nu^{k-1} E_{\nu-1}(f), n \in \mathbb{N}$, where $T_{n}(f) \equiv T_{n}(f ; x)$ is a trigonometric polynomial of best $C\left(\mathbb{T}\right.$ )-approximation to the function $f$ (i.e., $\left\|f-T_{n}(f)\right\|=E_{n}(f), n \in \mathbb{Z}_{+}$);

(c) the inverse theorem (with the derivatives), or the Vallée-Poussin-Stechkin inequality $\omega_{k}\left(f^{(r)} ; \pi / n\right) \leq$ $C_{8}(k, r)\left\{n^{-k} \sum_{\nu=1}^{n} \nu^{k+r-1} E_{\nu-1}(f)+\sum_{\nu=n+1}^{\infty} \nu^{r-1} E_{\nu-1}(f)\right\}$ for any $n \in \mathbb{N}$, as well as Stechkin's earlier inequality $E_{n-1}\left(f^{(r)}\right) \leq C_{9}(r)\left\{n^{r} E_{n-1}(f)+\sum_{\nu=n+1}^{\infty} \nu^{r-1} E_{\nu-1}(f)\right\}, \quad n \in \mathbb{N}$, where $E(f ; r) \equiv$ $\sum_{n=1}^{\infty} n^{r-1} E_{n-1}(f)<\infty$ (by S. N. Bernstein's theorem, this inequality guarantees that $f$ lies in $C^{r}(\mathbb{T}$ ), where $r \in \mathbb{N})$ can be supplemented with the following key inequalities: $\left\|f^{(r)}\right\| \leq C_{10}(r) E(f ; r)$ and $\left\|T_{n}^{(r)}(f)\right\| \leq$ $C_{7}(r) \sum_{\nu=1}^{n} \nu^{r-1} E_{\nu-1}(f), n \in \mathbb{N}$. Moreover, all the inequalities formulated in this paragraph are pairwise equivalent; i.e., any of these inequalities implies any other and, hence, all the inequalities.

Keywords: best approximation, modulus of smoothness, direct theorem, inverse theorem, order equality, equivalent inequalities, order-sharp inequality on a class.

MSC: 42A10, 41A17, 41A25, 42A32

DOI: $10.21538 / 0134-4889-2020-26-4-155-181$

\section{Введение}

Пусть $C(\mathbb{T})$ - пространство вещественнозначных непрерывных $2 \pi$-периодических функций с равномерной нормой $\|f\|=\max \{|f(x)|: x \in \mathbb{T}\}$, где $\mathbb{T}=(-\pi, \pi], C^{r}(\mathbb{T})-$ класс функций $f \in C(\mathbb{T})$, имеющих обычную производную $r$-го порядка $f^{(r)} \in C(\mathbb{T})$, где $r \in \mathbb{N}\left(f^{(0)} \equiv f\right.$, $\left.C^{0}(\mathbb{T}) \equiv C(\mathbb{T})\right)$

$E_{n}(f)$ - наилучшее равномерное приближение функции $f$ тригонометрическими полиномами порядка не выше $n, n \in \mathbb{Z}_{+}, T_{n}(f ; x)$ - полином наилучшего равномерного приближения функции $f$ порядка $n \in \mathbb{Z}_{+}:\left\|f(\cdot)-T_{n}(f ; \cdot)\right\|=E_{n}(f), n \in \mathbb{Z}_{+}$;

$\omega_{k}(f ; \delta)$ - модуль гладкости $k$-го порядка (при $k=1: \omega_{1}(f ; \delta) \equiv \omega(f ; \delta)$ - модуль непрерывности) функции $f \in C(\mathbb{T})$, где $k \in \mathbb{N}, \delta \in[0,+\infty): \omega_{k}(f ; \delta)=\sup \left\{\left\|\Delta_{h}^{k} f(\cdot)\right\|: h \in R,|h| \leq \delta\right\}$, $\Delta_{h}^{k} f(x)=\sum_{\nu=0}^{k}(-1)^{k-\nu}\left(\begin{array}{l}k \\ \nu\end{array}\right) f(x+\nu h),\left(\begin{array}{l}k \\ \nu\end{array}\right)=k ! /(\nu !(k-\nu) !), \nu=\overline{0, k}$.

Статья посвящена некоторым дополнениям и комментариям к неравенствам С. Б. Стечкина, полученным им при исследовании прямых и обратных теорем теории приближений непрерывных $2 \pi$-периодических функций и опубликованным в [1]. При этом особое внимание уделяется детальному обзору ранее полученных в этом направлении результатов.

Ниже приводятся формулировки указанных неравенств С.Б. Стечкина с дополнениями автора. Комментарии и доказательства соответствующих утверждений изложены в последующих разделах данной работы.

1. Прямая теорема, или неравенство Джексона - Стечкина (анонсировано в $[2$, п. 3, теорема 1], полное доказательство приведено в $[1, \S 2$, теорема 1 , неравенство (2.5)]):

$$
E_{n-1}(f) \leq C_{1}(k) \omega_{k}\left(f ; \frac{\pi}{n}\right), \quad n \in \mathbb{N} .
$$

Здесь и всюду в дальнейшем $C_{j}(k, r, \ldots)$, где $j \in \mathbb{N}$, обозначают положительные постоянные величины, зависящие только от указанных в скобках параметров.

Неравенство (0.1) допускает усиление (см. ниже разд. 1, подразд. 1.2).

Теорема 1. Пусть $f \in C(\mathbb{T}), k \in \mathbb{N}$, тогда

$$
\rho_{n}^{(k)}(f) \equiv n^{-k} \max _{1 \leq \nu \leq n}\left\{\nu^{k} E_{\nu-1}(f)\right\} \leq 2^{k} C_{1}(k) \omega_{k}\left(f ; \frac{\pi}{n}\right), \quad n \in \mathbb{N} .
$$

Усиление понимается в том смысле, что $E_{n-1}(f) \leq \rho_{n}^{(k)}(f), n \in \mathbb{N}$. Очевидно, что если $m^{k} E_{m-1}(f) \uparrow(m \uparrow)$, то $\rho_{n}^{(k)}(f)=E_{n-1}(f), n \in \mathbb{N}$. Справедливо также и обратное утверждение: если $\rho_{n}^{(k)}(f)=E_{n-1}(f), n \in \mathbb{N}$, то $m^{k} E_{m-1}(f) \uparrow(m \uparrow)$. 
Неравенства (0.1) и (0.2) являются точными в смысле порядка на классе всех функций $f \in C(\mathbb{T})$ с заданной мажорантой либо с заданным порядком убывания модуля гладкости $\omega_{k}(f ; \delta)$, а именно: для любых $k \in \mathbb{N}$ и $\omega \in \Omega_{k}(0, \pi]$ существует функция $f_{0}(\cdot ; \omega) \in C(\mathbb{T})$ (точнее, четная функция $f_{0}$ при нечетном $k$ и нечетная функция $f_{0}$ при четном $k$ ) такая, что $\omega_{k}\left(f_{0} ; \delta\right) \asymp C_{2}(k) \omega(\delta), \delta \in(0, \pi]$, при этом имеют место порядковые равенства (см. ниже разд. 1, подразд. 1.3)

$$
E_{n-1}\left(f_{0}\right) \asymp C_{3}(k) \rho_{n}^{(k)}\left(f_{0}\right) \asymp C_{4}(k) \omega_{k}\left(f_{0} ; \frac{\pi}{n}\right) \asymp C_{5}(k) \omega\left(\frac{\pi}{n}\right), \quad n \in \mathbb{N},
$$

где $\Omega_{k}(0, \pi]$ - класс функций $\omega=\omega(\delta)$, определенных на $(0, \pi]$ и удовлетворяющих условиям: $0<\omega(\delta) \downarrow 0(\downarrow 0)$ и $\delta^{-k} \omega(\delta) \downarrow(\delta \uparrow)$.

Вместе с тем существуют классы четных функций $f \in C(\mathbb{T})$ и нечетных функций $g \in$ $C(\mathbb{T})$ (при дополнительных условиях на поведение коэффициентов Фурье) такие, что неравенство (0.2) превращается в порядковое равенство (см. ниже разд. 1, подразд. 1.4.6)

$$
\rho_{n}^{(k)}(\psi) \asymp C_{6}(k)\left\{\omega_{k}\left(\psi ; \frac{\pi}{n}\right): \psi=f, k-\text { нечетное; } \psi=g, k-\text { четное }\right\}, \quad n \in \mathbb{N} .
$$

Напомним, что порядковое равенство $\varphi_{n} \asymp C_{7}(k, r, \ldots) \psi_{n}$ означает существование таких постоянных величин $0<C_{8} \leq C_{9}$, зависящих лишь от указанных в $C_{7}(k, r, \ldots)$ параметров $k, r, \ldots$, что $C_{8} \psi_{n} \leq \varphi_{n} \leq C_{9} \psi_{n}$. Если в $C_{7}$ не указаны параметры, то под $C_{8}$ и $C_{9}$ понимаются абсолютные постоянные величины и обозначение $C_{7}$ опускается.

Неравенство (0.1) допускает также усиление вида (см. ниже разд. 1, подразд. 1.4.2)

$$
\left(\prod_{\nu=1}^{n} E_{\nu-1}(f)\right)^{1 / n} \leq C_{10}(k) \omega_{k}\left(f ; \frac{\pi}{n}\right), \quad n \in \mathbb{N}
$$

Усиление понимается в том смысле, что величина $E_{n-1}(f)$ (в силу $\left.E_{m}(f) \downarrow(m \uparrow)\right)$ не превышает левую часть неравенства (0.5). Неравенство (0.5) также является точным в смысле порядка: для функции $f_{0}(\cdot ; \omega) \in C(\mathbb{T})$ в силу порядковых равенств $(0.3)$ имеем

$$
C_{5}(k) \omega\left(\frac{\pi}{n}\right) \leq\left(\prod_{\nu=1}^{n} E_{\nu-1}\left(f_{0}\right)\right)^{1 / n} \leq C_{10}(k) \omega_{k}\left(f_{0} ; \frac{\pi}{n}\right) \asymp C_{10}(k) C_{4}^{-1}(k) C_{5}(k) \omega\left(\frac{\pi}{n}\right), \quad n \in \mathbb{N} .
$$

Неравенство (0.2) сильнее неравенства (0.5), поскольку (см. ниже разд. 1, подразд. 1.4.3) справедлива оценка

$$
\left(\prod_{\nu=1}^{n} E_{\nu-1}(f)\right)^{1 / n}<e^{k} \rho_{n}^{(k)}(f) \equiv e^{k} n^{-k} \max _{1 \leq \nu \leq n}\left\{\nu^{k} E_{\nu-1}(f)\right\}, \quad n \in \mathbb{N} .
$$

Отметим, что наличие числа $e$ в оценке (0.6) связано с использованием известного из классического анализа неравенства $n^{n} e^{-n}<n !, n \in \mathbb{N}$.

Kроме того, уместно отметить также, что: $(0.1) \Leftrightarrow(0.2),(0.1) \Leftrightarrow(0.5)$ и $(0.2) \Leftrightarrow(0.5)$.

Выражение $\rho_{n}^{(k)}(f)$ в левой части неравенства $(0.2)$ не является самой "близкой слева" величиной к $\omega_{k}(f ; \pi / n)$, так как для любого $\beta \in[1, \infty)$ имеют место оценки (см. разд. 1 , подразд. 1.4.4)

$$
(\beta k)^{-1 / \beta} \rho_{n}^{(k)}(f) \leq n^{-k}\left(\sum_{\nu=1}^{n} \nu^{\beta k-1} E_{\nu-1}^{\beta}(f)\right)^{1 / \beta} \leq k^{1-1 / \beta} n^{-k} \sum_{\nu=1}^{n} \nu^{k-1} E_{\nu-1}(f), \quad n \in \mathbb{N},
$$

однако оценка сверху выражения в правой части $(0.7)$ посредством $\omega_{k}(f ; \pi / n)$ уже не имеет места на всем классе $C(\mathbb{T})$. 
Известным исключением являются классы четных функций $f \in C(\mathbb{T}$ ) (для четных значений $k$ ) и нечетных функций $g \in C(\mathbb{T})$ (для нечетных значений $k$ ) при дополнительных условиях на поведение коэффициентов Фурье, для которых имеют место порядковые равенства (см. ниже разд. 1, подразд. 1.4.6)

$$
n^{-k} \sum_{\nu=1}^{n} \nu^{k-1} E_{\nu-1}(\psi) \asymp C_{11}(k)\left\{\omega_{k}\left(\psi ; \frac{\pi}{n}\right): \psi=f, k \text { четное; } \psi=g, k \text { нечетное }\right\}, \quad n \in \mathbb{N} \text {. }
$$

Неравенство (0.2) можно дополнить следующей двусторонней оценкой (см. ниже разд. 1 , подразд. 1.4.5), представляющей также и самостоятельный интерес.

Теорема 2. Пусть $f \in C(\mathbb{T}), k \in \mathbb{N} ;$ тогда

$$
\left(C_{12}(k+1)\right)^{-1} \omega_{k+1}\left(f ; \frac{\pi}{n}\right) \leq \rho_{n}^{(k)}(f) \leq 2^{k} C_{1}(k) \omega_{k}\left(f ; \frac{\pi}{n}\right), \quad n \in \mathbb{N},
$$

где $C_{12}(k+1)=C_{14}(k+1)-$ постоянная величина в приведенном ниже неравенстве $(0.15)$.

Ясно, что неравенство слева в (0.9), вообще говоря, не имеет места, если $\rho_{n}^{(k)}(f)$ заменить на $E_{n-1}(f)$.

2. Оценка сверху нормы производной произвольного тригонометрического полинома посредством нормы конечной разности (доказательство опубликовано в [3], формулировка приведена также в $[1, \S 3$, теорема 2$]):$ пусть $k \in \mathbb{N}, 0<\delta<2 \pi / n$; тогда для произвольного полинома $t_{n}(x)$ порядка не выше $n$ имеет место неравенство

$$
\left\|t_{n}^{(k)}(\cdot)\right\| \leq n^{k}\left(2 \sin \frac{n \delta}{2}\right)^{-k}\left\|\Delta_{\delta}^{k} t_{n}(\cdot)\right\|, \quad n \in \mathbb{N},
$$

которое обращается в равенство в том и только том случае, если $t_{n}(x)=a \cos n x+b \sin n x+c$, где $a, b, c \in \mathbb{R}$.

Из (0.10) в качестве следствия выводится (см. $[1, \S 3$, следствие 2.2, неравенства (3.3)]) двусторонняя оценка

$$
\left(\frac{2}{n \delta} \sin \frac{n \delta}{2}\right)^{k} \leq \frac{\left\|\Delta_{\delta}^{k} t_{n}(\cdot)\right\|}{\delta^{k}\left\|t_{n}^{(k)}(\cdot)\right\|} \leq 1, \quad 0<\delta<\frac{2 \pi}{n}, \quad n \in \mathbb{N}
$$

Поскольку выражение в левой части (0.11) стремится к единице при $\delta \rightarrow 0$, то предел среднего члена в (0.11) при $\delta \rightarrow 0$ также равен единице, т. е. существует $\lim _{\delta \rightarrow+0}\left\|\delta^{-k} \Delta_{\delta}^{k} t_{n}(\cdot)\right\|=\left\|t_{n}^{(k)}(\cdot)\right\|$.

В случае $0<\delta \leq \pi / n$ оценке (0.11) можно придать более симметричную и точную в смысле порядка форму (см. ниже разд. 2, подразд. 2.3):

$$
\left(\frac{2}{n \delta} \sin \frac{n \delta}{2}\right)^{k} \leq \frac{\left\|\Delta_{\delta}^{k} t_{n}(\cdot)\right\|}{\delta^{k}\left\|t_{n}^{(k)}(\cdot)\right\|} \leq\left(\frac{\pi}{2}\right)^{k}\left(\frac{2}{n \delta} \sin \frac{n \delta}{2}\right)^{k} .
$$

Оценка в правой части $(0.11)$, которая следует из очевидного неравенства $\left\|\Delta_{\delta}^{k} t_{n}(\cdot)\right\| \leq$ $\delta^{k}\left\|t_{n}^{(k)}(\cdot)\right\|$, сильнее, чем оценка в правой части $(0.12)$, поскольку $\left(\frac{\pi}{2}\right)^{k}\left(\frac{2}{n \delta} \sin \frac{n \delta}{2}\right)^{k} \geq 1$. Однако (0.12) в силу симметричной формы часто удобнее в приложениях. Далее, поскольку $\left\|\Delta_{\delta}^{k} t_{n}(\cdot)\right\| \leq \omega_{k}\left(t_{n} ; \delta\right)$, то из неравенства (0.10) следует очевидная оценка (см. [1, §3, следствие 2.3, неравенство (3.5)])

$$
\left\|t_{n}^{(k)}(\cdot)\right\| \leq n^{k}\left(2 \sin \frac{n \delta}{2}\right)^{-k} \omega_{k}\left(t_{n} ; \delta\right), \quad 0<\delta \leq \frac{\pi}{n}, \quad n \in \mathbb{N}
$$

которую можно довести до порядкового равенства (см. ниже разд. 2, подразд. 2.4), т. е. справедливо следующее утверждение. 
Теорема 3. Пусть $k \in \mathbb{N}, 0<\delta \leq \pi / n, n \in \mathbb{N} ;$ тогда

$$
\omega_{k}\left(t_{n} ; \delta\right) \asymp C_{13}(k) \delta^{k}\left\|t_{n}^{(k)}(\cdot)\right\| .
$$

3. Обратная теорема (без производных), или неравенство Салема - Стечкина (см. $[1, \S 5$, теорема 8, неравенство (5.14)]): пусть $f \in C(\mathbb{T}), k \in \mathbb{N} ;$ тогда

$$
\omega_{k}\left(f ; \frac{\pi}{n}\right) \leq C_{14}(k) n^{-k} \sum_{\nu=1}^{n} \nu^{k-1} E_{\nu-1}(f), \quad n \in \mathbb{N} .
$$

Прежде всего отметим, что неравенство (0.1) можно дополнить следующими известными порядковыми равенствами (см. ниже разд. 3 , подразд. 3.1 .3 и 3.1.4):

$$
\begin{gathered}
\omega_{k}\left(f ; \frac{\pi}{n+1}\right) \asymp C_{15}(k)\left\{E_{n}(f)+(n+1)^{-k}\left\|T_{n}^{(k)}(f ; \cdot)\right\|\right\}, \quad n \in \mathbb{Z}_{+}, \\
\omega_{k}\left(f ; \frac{\pi}{n+1}\right) \asymp C_{16}(k)\left\|f(\cdot)-T_{n}(f ; \cdot)+(n+1)^{-k} T_{n}^{(k)}(f ; \cdot)\right\|, \quad n \in \mathbb{Z}_{+},
\end{gathered}
$$

которые в определенном смысле характеризуют порядок убывания модулей гладкости функций $f \in C(\mathbb{T})$ (в случае $n=0$ имеем $\left.\omega_{k}(f ; \pi) \asymp C_{17}(k) E_{0}(f)=C_{17}(k)\left\|f(\cdot)-T_{0}(f ; \cdot)\right\|\right)$.

Сопоставляя равенства $(0.16)$ и (0.17) приходим к порядковому равенству $\left(\left\|f(\cdot)-T_{n}(f ; \cdot)\right\|=\right.$ $\left.E_{n}(f), n \in \mathbb{Z}_{+}\right)$

$$
\begin{gathered}
\left\|f(\cdot)-T_{n}(f ; \cdot)\right\|+(n+1)^{-k}\left\|T_{n}^{(k)}(f ; \cdot)\right\| \\
\asymp C_{18}(k)\left\|f(\cdot)-T_{n}(f ; \cdot)+(n+1)^{-k} T_{n}^{(k)}(f ; \cdot)\right\|, \quad n \in \mathbb{Z}_{+} .
\end{gathered}
$$

Для доказательства неравенства (0.15) С. Б. Стечкиным впервые была применена ключевая оценка (см. $[1, \S 5$, лемма 10 , неравенство (5.10) и доказательство теоремы 8]): пусть $f \in C(\mathbb{T}), k \in \mathbb{N}$; тогда

$$
\left\|T_{n}^{(k)}(f ; \cdot)\right\| \leq C_{19}(k) \sum_{\nu=1}^{n} \nu^{k-1} E_{\nu-1}(f), \quad n \in \mathbb{N} .
$$

Привлечение (0.19) для доказательства (0.15), по всей видимости, связано с наличием оценки сверху в порядковом равенстве $(0.16)$ (см. $[1, \S 5$, доказательство леммы 9]), так что желание получить оценку сверху $\omega_{k}(f ; \pi / n)$ посредством лишь элементов последовательности $\left\{E_{n-1}(f)\right\}_{n=1}^{\infty}$ привело к необходимости доказательства оценки вида (0.19).

Исключительная роль оценки (0.19) подтверждается в следующей теореме (см. ниже разд. 3, подразд. 3.2).

Теорема 4. Пусть $f \in C(\mathbb{T}), k \in \mathbb{N} ;$ тогда для справедливости неравенства (0.15) необходимо и достаточно выполнение неравенства (0.19).

4. Обратная теорема с производными или неравенство Валле-Пуссена - Стечкина (см. $[1, \S 5$, теорема 10, неравенство (5.18) и следствие 10.1, неравенство (5.23); теорема 11, неравенство (5.24)]): пусть $f \in C(\mathbb{T}), k, r \in \mathbb{N}$ и $\sum_{n=1}^{\infty} n^{r-1} E_{n-1}(f)<\infty$; тогда $f \in C^{r}(\mathbb{T})$ и справедливы неравенства

$$
\begin{gathered}
E_{n-1}\left(f^{(r)}\right) \leq C_{20}(r)\left\{n^{r} E_{n-1}(f)+\sum_{\nu=n+1}^{\infty} \nu^{r-1} E_{\nu-1}(f)\right\}, \quad n \in \mathbb{N}, \\
\omega_{k}\left(f^{(r)} ; \frac{\pi}{n}\right) \leq C_{21}(k, r)\left\{n^{-k} \sum_{\nu=1}^{n} \nu^{k+r-1} E_{\nu-1}(f)+\sum_{\nu=n+1}^{\infty} \nu^{r-1} E_{\nu-1}(f)\right\}, \quad n \in \mathbb{N} .
\end{gathered}
$$


Неравенства (0.20) и (0.21) можно также дополнить ключевой оценкой

$$
\left\|f^{(r)}\right\| \leq C_{22}(r) \sum_{n=1}^{\infty} n^{r-1} E_{n-1}(f) \equiv C_{22}(r) E(f ; r)
$$

из которой сразу следует (0.20), а также (0.21) (т. е. обратная теорема с производными), если привлечь неравенство (0.19) со значением $k+r$ вместо $k$, выполнение которого не только достаточно, но и необходимо для справедливости (0.21) (см. ниже замечание 2 в разд. 4 , подразд. 4.5).

На самом деле имеют место более сильные утверждения (см. ниже разд. 4, подразд. 4.3-4.6).

Теорема 5. Пусть $f \in C(\mathbb{T}), r \in \mathbb{N} u E(f ; r)<\infty$. Для справедливости неравенства

$$
\left\|T_{n}^{(r)}(f ; \cdot)\right\| \leq C_{19}(r) \sum_{\nu=1}^{n} \nu^{r-1} E_{\nu-1}(f), \quad n \in \mathbb{N}
$$

необходимо и достаточно выполнение равенства (0.22).

Теорема 6. Пусть $f \in C(\mathbb{T}), r \in \mathbb{N} u E(f ; r)<\infty$. Для справедливости неравенства $(0.20)$ необходимо и достаточно выполнение неравенства (0.22).

Теорема 7. Пусть $f \in C(\mathbb{T}), k, r \in \mathbb{N} u E(f ; r)<\infty$. Для справедливости неравенства (0.20) необходимо и достаточно выполнение неравенства (0.21).

Теорема 8. Пусть $f \in C(\mathbb{T}), k, r \in \mathbb{N} u E(f ; r)<\infty$. Для справедливости неравенства (0.21) необходимо и достаточно выполнение неравенства (0.22).

Таким образом, подводя итог вышеизложенному, заметим в плане оформления текста статьи следующее: по каждому из выписанных в пп. 1-4 неравенств имеется так называемая "история вопроса", которую мы отсылаем в соответствующие разделы, чтобы не нагружать потоками ссылок на ранние, либо поздние работы и так переполненное по объему введение.

\section{1. Прямая теорема}

\section{1. Исторический комментарий к неравенству (0.1)}

При изложении этого подраздела автор стремился, основываясь только на оригинальных источниках, проследить историю вопроса до появления неравенства (0.1) в работе С. Б. Стечкина [2], полное доказательство которого позднее было опубликовано в [1].

Вначале отметим, что в силу известного свойства модуля гладкости $\omega_{k+r}(f ; \delta) \leq$ $\delta^{r} \omega_{k}\left(f^{(r)} ; \delta\right)$, где $r \in \mathbb{N}, f \in C^{r}(\mathbb{T})$, из $(0.1)$ следует оценка $E_{n-1}(f) \leq C_{1}(k+r) \omega_{k+r}(f ; \pi / n) \leq$ $C_{1}(k+r) \pi^{r} n^{-r} \omega_{k}\left(f^{(r)} ; \pi / n\right), n \in \mathbb{N}$, откуда, учитывая также $(0.1)$, имеем (см. $[1, \S 2$, следствие 1.1, неравенство (2.7)]):

$$
E_{n-1}(f) \leq C_{1}(k+r) \pi^{r} n^{-r} \omega_{k}\left(f^{(r)} ; \frac{\pi}{n}\right), \quad f \in C^{r}(\mathbb{T}), r \in \mathbb{Z}_{+}, k \in \mathbb{N}, n \in \mathbb{N} .
$$

Таким образом, все неравенства с производными $f^{(r)}$ порядка $r \in \mathbb{N}\left(f^{(0)} \equiv f\right)$ в правой части (1.1) являются следствиями (0.1).

Кроме того, в силу известного неравенства $\omega_{r}(f ; \delta) \leq \delta^{r}\left\|f^{(r)}\right\|$, где $r \in \mathbb{N}, f \in C^{r}(\mathbb{T})$, из (0.1) следует оценка $E_{n-1}(f) \leq C_{1}(r) \pi^{r} n^{-r}\left\|f^{(r)}\right\|, n \in \mathbb{N}$. Очевидно, что последняя оценка также следует из неравенства (1.1), поскольку в этом случае $\omega_{k}\left(f^{(r)} ; \delta\right) \leq 2^{k}\left\|f^{(r)}\right\|$. 
Из неравенства (1.1) следуют все перечисленные ниже утверждения, и поэтому как для полноты изложения, так и в силу наличия разночтений в монографической литературе, возникает естественная необходимость в детальном напоминании ранее полученных результатов также и при $r \in \mathbb{N}$. Как оказалось, существенные технические трудности возникали при доказательстве неравенств вида (1.1) именно в случае $r \in \mathbb{N}$, поскольку оценки уклонения заданной функции от полинома приближения фактически производились внутри интегральных представлений, содержащих первые разности либо симметрические разности второго порядка.

Неравенства вида (1.1) при $k=1$ называют неравенствами Джексона, а при $k>1-$ неравенствами Джексона-Стечкина.

В литературе по теории приближений, как правило, широко освещаются теоремы (либо неравенства) Джексона $(1911,1930)$, при этом авторы позволяют себе придавать им ту или иную, иногда весьма вольную, но выгодную для изложения формулировку. Вместе с тем, очень мало внимания уделяется результатам Валле-Пуссена (1919), который внес существенный вклад в усовершенствование методов доказательства Джексона, значительно упростив при этом технические выкладки в случае $r \in \mathbb{N}$.

1.1.1. Неравенства (0.1) для различных значений $k \in \mathbb{N}$ непосредственно следуют из оценок вида

$$
\left\|f(\cdot)-t_{n-1}(\cdot)\right\| \leq C_{1}(k) \omega_{k}(f ; \pi / n), \quad n \in \mathbb{N},
$$

где $t_{n-1}(x)$ представляет собой, как правило, свертку заданной функции с некоторым специально подобранным полиномиальным ядром.

В случае $k=1$ подобная оценка была впервые установлена Д. Джексоном [4, гл. II; разд. 3, теорема VIII, с. 48] в 1911 году. Первая публикация в открытой печати [5] появилась в 1912 году, где, в частности, была приведена ключевая теорема с полным доказательством [5, разд. I, теорема I, с. 492] (см. также [4, гл. II, разд. 1, теорема VI, с. 42; 6, гл. I, разд. 1, теорема I, с. 2]) для случая функций, удовлетворяющих условию Липшица, а именно: если $f \in C(\mathbb{T}) u \omega(f ; \delta)=$ $O(\delta), \delta \in(0,+\infty)$, то для каждого $n \in \mathbb{N}$ существует полином $t_{n}(x)$ порлдка не выше $n$ такой, что $\left\|f(\cdot)-t_{n}(\cdot)\right\|=O\left(n^{-1}\right), n \in \mathbb{N}$. Общий случай функций $f \in C(\mathbb{T})$ с заданным модулем непрерывности $\omega(f ; \delta)$, применением рассуждений геометрического характера (см. [6, гл. I, разд. 1, теорема II, доказательство на с. 7], а также [4, гл. II, разд. 3, теорема VIII, c. 48]), сводится к утверждению теоремы I [5, разд. I; 6, гл. I, разд. 1], либо теоремы VI [4, гл. II, разд. 1]:

$$
\left\|f(\cdot)-t_{n}(\cdot)\right\|=O\left(\omega\left(f ; n^{-1}\right)\right), \quad n \in \mathbb{N} .
$$

В этих же работах [4, гл. II, разд. 2, теорема VII, с. 46; 5, разд. I, теорема III, с. 496; 6, гл. I, разд. 1, теорема III, с. 10] дано обобщение (точнее, установлен аналог) теоремы I [5, разд. I; 6 , гл. I, разд. 1] или, что то же самое, теоремы VI [4, гл. II, разд. 1], на случай функций $f \in C^{r-1}(\mathbb{T}), r \in \mathbb{N}$, c $\omega\left(f^{(r-1)} ; \delta\right)=O(\delta), \delta \in(0,+\infty)$; соответствующая оценка выглядит следующим образом:

$$
\left\|f(\cdot)-t_{n}(\cdot)\right\|=O\left(n^{-r}\right), \quad n \in \mathbb{N} .
$$

Следует отметить, что методы доказательства последней оценки, приведенные в указанных выше работах, существенно отличаются как в идейном плане, так и в техническом исполнении. Случай функций $f \in C^{r}(\mathbb{T})$ с модулем непрерывности $\omega\left(f^{(r)} ; \delta\right)$ анонсирован в [6, гл. I, разд. 1 , теорема IV]:

$$
\left\|f(\cdot)-t_{n}(\cdot)\right\|=O\left(n^{-r} \omega\left(f^{(r)} ; 1 / n\right)\right), \quad n \in \mathbb{N}
$$

при этом там же было указано, что для установления последнего утверждения может быть применен тот же самый процесс индукции, который был использован при доказательстве предыдущей теоремы III [6, гл. I, разд. 1].

\subsection{2. Обозначим:}


$F_{m}(u)=\left(\frac{\sin m u}{m \sin u}\right)^{4}, \quad I_{m}(x)=\frac{1}{\gamma_{m}} \int_{-\pi / 2}^{\pi / 2} f(x+2 u) F_{m}(u) d u, \quad$ где $\quad \gamma_{m}=\int_{-\pi / 2}^{\pi / 2} F_{m}(u) d u, \quad m \in \mathbb{N}$.

Тогда имеют место равенства (см. [6, гл. I, разд. 1, доказательство теоремы I, с. 3-4; 5, разд. I, доказательство теоремы I, с. 492, доказательство теоремы III, с. 496; 4, гл. II, разд. 1, доказательство теоремы VI, с. 43])

$$
\begin{aligned}
& I_{m}(x)-f(x)=\gamma_{m}^{-1} \int_{-\pi / 2}^{\pi / 2}[f(x+2 u)-f(x)] F_{m}(u) d u \\
& =2 \gamma_{m}^{-1} \int_{-\pi / 2}^{\pi / 2}[f(x+2 u)-2 f(x)+f(x-2 u)] F_{m}(u) d u .
\end{aligned}
$$

Функция $I_{m}(x)$ представляет собой полином порядка $2(m-1)$, где $m \in \mathbb{N}$. Для каждого $n \in \mathbb{N}$, полагая (см. [6, гл. I, разд. 1, доказательство теоремы I, с. 6]) $m=(1 / 2) n+1$ при четном $n$ и $m=(1 / 2)(n+1)$ при нечетном $n$, имеем $2(m-1) \leq n<2 m(\Rightarrow 1 / m<2 / n)$. Так что, обозначая выражение $I_{m}(x)$ через $t_{n}(x)$, получаем, что $t_{n}(x)$ является полиномом порядка не выше $n$. Другой вариант перехода от $I_{m}(x)$ к $t_{n}(x)$ предложен также в [4, гл. II, разд. 1, с. $45 ; 5$, гл. I, разд. I, доказательство теоремы I, с. 494].

Д. Джексон [4, гл. II, разд. 1, доказательство теоремы VI, с. 43-44; 5, разд. 1, доказательство теоремы I, с. 493-494; 6, гл. I, разд. 1, доказательство теоремы I, с. 4-6] показал, что, учитывая в левом равенстве для $I_{m}(x)-f(x)$ условие Липшица $|f(x+h)-f(x)|=O(|h|), x, h \in \mathbb{R}$, получаем оценку

$$
\left\|t_{n}(\cdot)-f(\cdot)\right\|=O\left(n^{-1}\right), \quad n \in \mathbb{N} .
$$

А. Зигмунд [7, разд. 5, доказательство теоремы 8 в части “достаточность", с. 61-62] отметил, что если в правом равенстве для $I_{m}(x)-f(x)$ применить оценку $|f(x+h)-2 f(x)+f(x+h)|=$ $O(|h|), x, h \in \mathbb{R}$, то также получим $\left\|t_{n}(\cdot)-f(\cdot)\right\|=O\left(n^{-1}\right), n \in \mathbb{N}$. Таким образом, А. Зигмундом установлена справедливость импликации

$$
\omega_{2}(f ; \delta)=O(\delta), \quad \delta \in[0,+\infty) \Rightarrow E_{n-1}(f)=O\left(n^{-1}\right), \quad n \in \mathbb{N} .
$$

1.1.3. В связи с вышеизложенным, уместно напомнить о соответствующих результатах Ш. Валле-Пуссена [8, гл. III]. Положим ([8, гл. III, разд. 31])

$$
G_{s}(t)=\left(\frac{\sin t}{t}\right)^{2 s}, \quad s \in \mathbb{N}, \quad V_{s}(x ; n)=\frac{1}{\tau(s)} \int_{-\infty}^{+\infty} f\left(x+\frac{2 t}{n}\right) G_{s}(t) d t, \quad n \in \mathbb{N}
$$

где $\tau(s)=\int_{-\infty}^{+\infty} G_{s}(t) d t$. Функция $V_{s}(x ; n)$ является тригонометрическим полиномом порядка меньше $s n$. Поскольку $\tau(2)=2 \pi / 3$, то $V_{2}(x ; n)=(3 / 2 \pi) \int_{-\infty}^{+\infty} f(x+2 t / n) G_{2}(t) d t$ (см. [8, гл. III, разд. 32]), причем порядок полинома $V_{2}(x ; n)$ равен $2 n-1$. В этом случае имеют место представления (см. [8, гл. III, разд. 33 и 34])

$$
V_{2}(x ; n)-f(x)=\frac{3}{2 \pi} \int_{-\infty}^{+\infty}\left[f\left(x+\frac{2 t}{n}\right)-f(x)\right] G_{2}(t) d t
$$




$$
=\frac{3}{2 \pi} \int_{0}^{+\infty}\left[f\left(x+\frac{2 t}{n}\right)+f\left(x-\frac{2 t}{n}\right)-2 f(x)\right] G_{2}(t) d t .
$$

Используя равенства (1.2), Ш. Валле-Пуссен привел прямые доказательства следующих утверждений, уточняющих, в частности, границы значений постоянных величин в соответствующих неравенствах Д. Джексона (см. подразд. 1.1.1):

a) если $f \in C(\mathbb{T})$, то $\left\|V_{2}(\cdot ; n)-f(\cdot)\right\|<C_{23} \omega(f ; 1 / n), n \in \mathbb{N}$, где $C_{23}<1+6 / \pi<3$ (см. $[8$, гл. III, разд. 33 , теорема I]);

b) если $f \in C^{1}(\mathbb{T})$, то $\left\|V_{2}(\cdot ; n)-f(\cdot)\right\|<C_{24} n^{-1} \omega\left(f^{\prime} ; 1 / n\right), n \in \mathbb{N}$, где $C_{24}<(3 / \pi)(\pi / 2+1)<$ $5 / 2$ (см. [8, гл. III, разд. 34 , теорема III]).

Кроме того, в этой же работе Ш. Валле-Пуссен значительно усовершенствовал методы доказательства неравенств (1.1) с производными порядка $r \in \mathbb{N}$ (см. [8, гл. III, разд. 36, теорема III, с. 48-50]), установив при этом справедливость следующих теорем:

c) если $r \in \mathbb{N}, f \in C^{r}(\mathbb{T})$ и $\left\|f^{(r)}\right\|<C_{25}(r)$, то существует полином $t_{m}(x)$ порядка не выше $m \in \mathbb{N}$ такой, что $\left\|t_{m}(\cdot)-f(\cdot)\right\|<C_{26}(r) C_{25}(r) m^{-r}, m \in \mathbb{N}$ (см. [8, гл. III, разд. 37, теорема III bis., c. 50]);

d) если $r \in \mathbb{N}, f \in C^{r}(\mathbb{T})$, то $\left\|t_{m}(\cdot)-f(\cdot)\right\|<C_{27}(r) m^{-r} \omega\left(f^{(r)} ; \pi / m\right), m \in \mathbb{N}$ (см. [8, гл. III, разд. 38, теорема IV, с. 51]);

е) если $r \in \mathbb{N}, f \in C^{r}(\mathbb{T})$ и $\omega\left(f^{(r)} ; \delta\right)=O\left(\delta^{\alpha}\right)$, где $0<\alpha \leq 1$, то $\left\|t_{m}(\cdot)-f(\cdot)\right\|<$ $C_{28}(r, \alpha) m^{-(r+\alpha)}, m \in \mathbb{N}$ (см. [8, гл. III, разд. 38 , теорема на с. 52]).

Следует также отметить, что если во втором из равенств (1.2) применить неравенство $|f(x+2 t / n)-2 f(x)+f(x-2 t / n)| \leq \omega_{2}(f ; 2 t / n) \leq(2 t+1)^{2} \omega_{2}(f ; 1 / n)$, то получим оценку

$$
\left|V_{2}(x ; n)-f(x)\right| \leq\left(\frac{3}{2 \pi}\right) \omega_{2}\left(f ; \frac{1}{n}\right) \int_{0}^{+\infty}(2 t+1)^{2} G_{2}(t) d t=O\left(\omega_{2}\left(f ; \frac{1}{n}\right)\right), \quad n \in \mathbb{N}
$$

(см. также [9, 1-е изд., Примечания, $n^{\circ}$ 75; 2-е изд., Дополнения и задачи, разд. III, п. 89]).

1.1.4. Н. И. Ахиезер [9, 1-е изд., гл. V, $n^{\circ}$ 87; 2-е изд., гл. V, $n^{\circ}$ 98], ссылаясь на работы [4; 6], отметил, что Д. Джексон доказал следующее важное предложение: если $f \in C^{r}(\mathbb{T})$, где $r \geq 1 u\left\|f^{(r)}\right\| \leq 1$, то при любом $n \in \mathbb{N}$ существует полином $t_{n-1}(x)$, удовлетворяющий неравенству $\left|f(x)-t_{n-1}(x)\right| \leq C_{29}(r) n^{-r}, x \in \mathbb{T}$. На самом деле в цитируемых работах такое предложение отсутствует, но оно непосредственно следует из утверждения теоремы IV [6, гл. I, разд. 1, с. 12] в силу очевидного неравенства $\omega\left(f^{(r)} ; \delta\right) \leq 2\left\|f^{(r)}\right\|$. Отметим также, что указанное предложение имеется в работе Валле-Пуссена [8] (см. выше подразд. 1.1.3, теорема $c)$ ).

С помощью теоремы 1 [9, 1-е изд., гл. V, $n^{\circ}$ 87; 2-е изд., гл. V, $\left.n^{\circ} 101\right]$, которая представляет собой уточнение (в смысле значения постоянной величины $C_{29}(r)$ ) сформулированного выше "предложения" Джексона, и имеет место в случае $r=0$, а также привлекая функции Стеклова (см. $\left[9,1\right.$-е изд., гл. V, $n^{\circ} 83 ; 2$-е изд., гл. V, $n^{\circ}$ 95]), Н. И. Ахиезер устанавливает $[9,1$-е изд., гл. V, $n^{\circ} 89 ; 2$-е изд., гл. V, $\left.n^{\circ} 105\right]$ справедливость неравенств:

$$
E_{n-1}(f) \leq C_{30} n^{-r} \omega\left(f^{(r)} ; 1 / n\right) \quad \text { и } E_{n-1}(f) \leq C_{31} n^{-r} \omega_{2}\left(f^{(r)} ; 1 / n\right),
$$

где $C_{30}=3, r \in \mathbb{Z}_{+}, n \in \mathbb{N}$. Из второго неравенства при $r=0$ следует $(0.1)$ в случае $k=2$. Метод доказательства Н. И. Ахиезера неравенства (0.1) в случае $k=2$ не является конструктивным, поскольку явно не выписывается аппроксимирующий полином, равномерное уклонение которого от заданной функции в последствии оценивается сверху посредством модуля 
непрерывности второго порядка (см. по этому поводу также [10, гл. V, п. 5.5.4, гл. III, п. 3.12.1]). Следует отметить, что позднее последние два неравенства тем же методом были заново установлены А. Ф. Тиманом [10, гл. V, п. 5.5.4]:

$$
E_{n-1}(f)<3 n^{-r} \omega\left(f^{(r)} ; 1 / n\right) \text { и } E_{n-1}(f)<(5 / 2) n^{-r} \omega_{2}\left(f^{(r)} ; 1 / n\right),
$$

и которые указывают на одно из возможных значений постоянных величин $C_{30}$ и $C_{31}$. Интересно заметить, что в приведенных выше результатах Валле-Пуссена [8] (см. подразд. 1.1.3, утв. $a)$ и $b)$ ) имеют место такие же ограничения: $C_{23}<3$ и $C_{24}<5 / 2$.

Из результатов, полученных С.Н. Бернштейном [11, следствие 5], следует справедливость утверждения (в указанной заметке явно не выписано): если $f \in C(\mathbb{T}) u \omega_{k}(f ; \delta)=O\left(\delta^{\alpha}\right)$, $\delta \in[0,+\infty)$, где $k \in \mathbb{N}, 0<\alpha \leq k$, mо $E_{n-1}(f)=O\left(n^{-\alpha}\right), n \in \mathbb{N}$.

В связи с этим утверждением заметим, что идея привлечения конечных разностей высших порядков к доказательству неравенств вила (1.1), но с производными порядка $r \in \mathbb{N}$ в правых частях, отмечена значительно ранее в диссертации Д. Джексона [4, гл. I, разд. 3, с. 28-30].

Полная реализация соответствующего метода была осуществлена в [5, разд. I, с. 497-500], где приводится другое доказательство теоремы III [5, разд. I, с. 496]. Указанное доказательство основано на оценке модуля представления (см. выше подразд. 1.1.2) для $I_{m}(x)-f(x)$, где в подынтегральном выражении выписана конечная разность $r$-го порядка функции $f$ в точке $x$ с шагом $2 u$, а показатель, равный 4 , в определении $F_{m}(u)$ заменен на величину $2 æ$, при этом

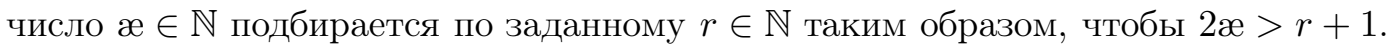

Ключевые оценки (см. [5, разд. I, с. 499], а также [4, гл. I, разд. 2, с. 25-26])

$$
\int_{0}^{\pi / 2} u^{r}\left(\frac{\sin m u}{m \sin u}\right)^{2 æ} d u<C_{32}(r, æ) m^{-(r+1)}, \quad \int_{0}^{\pi / 2} u^{r}\left(\frac{\sin m u}{m \sin u}\right)^{2 æ} d u>C_{33}(æ) m^{-1},
$$

гарантируют выполнение неравенства $\left\|I_{m}(\cdot)-f(\cdot)\right\|=O\left(m^{-r}\right), m \in \mathbb{N}$. В рассматриваемом случае порядок полинома $I_{m}(x)$ не превышает $(m-1)$, но процедура перехода, приведенная в подразд. 1.1.2, позволяет сформулировать последнюю оценку в виде $\left\|t_{n}(\cdot)-f(\cdot)\right\|=O\left(n^{-r}\right)$, $n \in \mathbb{N}$.

Очевидно, что этот же метод годится для получения неравенства вида (1.1) в случае $r=0$ и $\omega_{k}(f ; \delta)=O\left(\delta^{k}\right)$ (см. оценки $\left.(1.3)\right): E_{n-1}(f)=O\left(n^{-k}\right), n \in \mathbb{N}$. Позднее сформулированная выше идея и соответствующий метод были подтверждены также А. Зигмундом [7, разд. 5 , замечание $(i i)$, с. 64], причем там же было указано, что использование интегралов Джексона высших порядков не создает трудностей при доказательстве и позволяет установить справедливость следующих утверждений:

(а) если $f \in C(\mathbb{T}), r \in \mathbb{N}$ и $\omega_{r+2}(f ; \delta)=O\left(\delta^{r+1}\right)$, то $f \in C^{r}(\mathbb{T})$ и $\omega_{2}\left(f^{(r)} ; \delta\right)=O(\delta)$, откуда в силу теоремы $9[7$, п. 1 , с. 52 , доказательство приведено в п. 5 на с. 62$]$ имеем $E_{n}(f)=$ $O\left(n^{-(r+1)}\right), n \in \mathbb{N}$;

(b) если $f \in C(\mathbb{T}), r \in \mathbb{N}, 0<\alpha<1$ и $\omega_{r+1}(f ; \delta)=O\left(\delta^{r+\alpha}\right)$, то $f \in C^{r}(\mathbb{T})$ и $\omega\left(f^{(r)} ; \delta\right)=O\left(\delta^{\alpha}\right)$, и, следовательно, в силу теоремы IV [8, гл. III, разд. $38 ; 6$, гл. I, разд. 1] получаем $E_{n}(f)=O\left(n^{-(r+\alpha)}\right), n \in \mathbb{N}$.

\section{2. Доказательство теоремы 1}

Применяя неравенство (0.1) и учитывая известное свойство модуля гладкости (см., например, $\left[1, \S 1\right.$, лемма 5, неравенство (1.7)]) $\delta_{2}^{-k} \omega_{k}\left(f ; \delta_{2}\right) \leq 2^{k} \delta_{1}^{-k} \omega_{k}\left(f ; \delta_{1}\right)$, где $0<\delta_{1}<\delta_{2}<+\infty$, имеем

$$
\nu^{k} E_{\nu-1}(f) \leq C_{1}(k) \nu^{k} \omega_{k}(f ; \pi / \nu) \leq C_{1}(k) 2^{k} n^{k} \omega_{k}(f ; \pi / n), \quad 1 \leq \nu \leq n,
$$

откуда следует неравенство (0.2): 


$$
\rho_{n}^{(k)}(f) \equiv n^{-k} \max \left\{\nu^{k} E_{\nu-1}(f): 1 \leq \nu \leq n\right\} \leq 2^{k} C_{1}(k) \omega_{k}\left(f ; \frac{\pi}{n}\right) .
$$

3 а м е ч а н и е 1.

1) Оценка $\rho_{n}^{(k)}(f) \geq E_{n-1}(f), n \in \mathbb{N}$, имеет место в силу $E_{\nu-1}(f) \downarrow(\nu \uparrow)$.

$2)$ Если $\nu^{k} E_{\nu-1} \uparrow(\nu \uparrow)$, то очевидно, что $\rho_{n}^{(k)}(f)=E_{n-1}(f), n \in \mathbb{N}$. С другой стороны, если $\rho_{n}^{(k)}(f)=E_{n-1}(f), n \in \mathbb{N}$, то $\max \left\{\nu^{k} E_{\nu-1}(f): 1 \leq \nu \leq n\right\}=n^{k} E_{n-1}(f)$, откуда $n_{1}^{k} E_{n_{1}-1}(f)=\max \left\{\nu^{k} E_{\nu-1}(f): 1 \leq \nu \leq n_{1}\right\} \leq \max \left\{\nu^{k} E_{\nu-1}(f): 1 \leq \nu \leq n_{2}\right\}=n_{2}^{k} E_{n_{2}-1}(f)$ для любых $n_{1}, n_{2} \in \mathbb{N}, n_{1}<n_{2}$, и, следовательно, $n^{k} E_{n-1}(f) \uparrow(n \uparrow)$.

\section{3. Доказательство порядковых равенств (0.3)}

Нам понадобится следующая лемма, которая является, по существу, следствием соответствующих результатов В.Э.Гейта [12]. Поскольку утверждение этой леммы явно не простматривается в [12], а также в силу ее важности для справедливости (0.3), ниже приводится доступное доказательство.

Лемма 1. Пусть $k \in \mathbb{N}, \omega \in \Omega_{k}(0, \pi] ;$ существует функиия $f_{0}(x ; \omega) \in C(\mathbb{T})$ такал, что:

1) $\omega_{k}\left(f_{0} ; \delta\right) \asymp C_{34}(k) \omega(\delta), \quad \delta \in(0, \pi]$;

2) $E_{n-1}\left(f_{0}\right) \asymp C_{35}(k) \omega(\pi / n), \quad n \in \mathbb{N}$.

Д о к а з а т е л ь с т в о. Достаточно доказать оченку сверху в п. 1$): \omega_{k}\left(f_{0} ; \delta\right) \leq C_{36}(k) \omega(\delta)$, $\delta \in(0, \pi]$, и оценку снизу в $n .2): E_{n-1}\left(f_{0}\right) \geq C_{37}(k) \omega(\pi / n), n \in \mathbb{N}$, поскольку в этом случае с учетом неравенства (0.1) справедливы также оценки

$$
\omega\left(\frac{\pi}{n}\right) \leq C_{37}^{-1}(k) E_{n-1}\left(f_{0}\right) \leq C_{37}^{-1}(k) C_{1}(k) \omega_{k}\left(f_{0} ; \frac{\pi}{n}\right) \leq C_{37}^{-1}(k) C_{1}(k) C_{36}(k) \omega\left(\frac{\pi}{n}\right), \quad n \in \mathbb{N} .
$$

Из цепочки неравенств (1.4) следуют оценка снизу в $n .1)$ :

$$
\omega(\pi / n) \leq C_{37}^{-1}(k) C_{1}(k) \omega_{k}\left(f_{0} ; \pi / n\right), \quad n \in \mathbb{N} \Rightarrow \omega(\delta) \leq 2^{k} C_{37}^{-1}(k) C_{1}(k) \omega_{k}\left(f_{0} ; \delta\right), \quad \delta \in(0, \pi],
$$

и оценка сверху в $n .2)$ :

$$
E_{n-1}\left(f_{0}\right) \leq C_{1}(k) C_{36}(k) \omega(\pi / n), \quad n \in \mathbb{N} .
$$

Для заданной функции $\omega(\delta) \in \Omega_{k}(0, \pi]$ обозначим $\omega_{n}=\omega(\pi / n), \quad \Delta \omega_{n}=\omega_{n}-\omega_{n+1}$, $n \in \mathbb{N}$. При четном $k$ полагаем $f_{0}(x ; \omega)=f_{1}(x ; \omega)$, где (см. $[12, \S 3$, доказательство леммы 4 , формула (28)]; там же приведены соответствующие ссылки)

$$
f_{1}(x ; \omega)=\sum_{n=1}^{\infty} n^{-1} \omega_{n} \sin n x=\sum_{n=1}^{\infty} \Delta \omega_{n} \sum_{\nu=1}^{n} \nu^{-1} \sin \nu x .
$$

Очевидно, что указанные ряды равномерно сходятся всюду на $\mathbb{R}$ к своей сумме $f_{1}(x ; \omega)$, так что $f_{1} \in C(\mathbb{T})$ и синус-коэффициенты Фурье $b_{n}\left(f_{1}\right)=n^{-1} \omega_{n}, n \in \mathbb{N}$.

Поскольку (см. [12, §3, неравенство (30)]) $\left\|f_{1}-S_{n}\left(f_{1}\right)\right\| \leq 2 C_{38} \omega_{n+1}, n \in \mathbb{Z}_{+}$, где $S_{n}(\psi) \equiv S_{n}(\psi ; x)$ - частная сумма порядка $n$ ряда Фурье функции $\psi \in C(\mathbb{T}), C_{38}=$ $\sup \left\{\left\|\sum_{\nu=1}^{n} \nu^{-1} \sin \nu x\right\|: n \in \mathbb{N}\right\}<\infty$, то в силу неравенства (5) [12, § 2, лемма 1] с учетом условия $\delta^{-k} \omega(\delta) \downarrow(\delta \uparrow)\left(\Rightarrow n^{k} \omega_{n} \uparrow(n \uparrow)\right)$ имеем

$$
\omega_{k}\left(f_{1} ; \frac{\pi}{n+1}\right) \leq \pi^{k} C_{38}\left\{(n+1)^{-k} \sum_{\nu=1}^{n}\left|\Delta\left(\nu^{k+1} b_{\nu}\left(f_{1}\right)\right)\right|+(n+1) b_{n+1}\left(f_{1}\right)\right\}+2^{k}\left\|f_{1}-S_{n+1}\left(f_{1}\right)\right\|
$$


$\leq \pi^{k} C_{38}\left\{(n+1)^{-k} \sum_{\nu=1}^{n}\left[(\nu+1)^{k} \omega_{\nu+1}-\nu^{k} \omega_{\nu}\right]+\omega_{n+1}\right\}+2^{k+1} C_{38} \omega_{n+1} \leq 2 C_{38}\left(\pi^{k}+2^{k}\right) \omega_{n+1}, \quad n \in \mathbb{N} ;$ откуда $\omega_{k}\left(f_{1} ; \pi /(n+1)\right) \leq C_{39}(k) \omega(\pi /(n+1))$, и, следовательно, $\omega_{k}\left(f_{1} ; \pi / n\right) \leq 2^{k} C_{39}(k) \omega(\pi / n)$, $n \in \mathbb{N}$. Из последнего неравенства следует оченка сверху в $n .1)$ для случая четного $k$ :

$$
\omega_{k}\left(f_{1} ; \delta\right) \leq 2^{2 k} C_{39}(k) \omega(\delta), \quad \delta \in(0, \pi] .
$$

Для доказательства оценки снизу в п. 2) обозначим через $F_{1}$ первообразную функции $f_{1}$. Поскольку функция $-F_{1} \in C(\mathbb{T})$ является четной с положительными косинус-коэффициентами Фурье $a_{n}\left(-F_{1}\right)=n^{-2} \omega_{n}, n \in \mathbb{N}$, то в силу известного неравенства Н. К. Бари (см., например, $\left[12, \S 3\right.$, неравенство (33)]) и условия $n^{k} \omega_{n} \uparrow(n \uparrow)$ получаем

$$
\begin{gathered}
E_{n-1}\left(F_{1}\right)=E_{n-1}\left(-F_{1}\right) \geq E_{n}\left(-F_{1}\right) \geq 4^{-1} \sum_{\nu=2 n}^{\infty} \nu^{-2} \omega_{\nu} \geq 4^{-1} \sum_{\nu=2 n+1}^{3 n} \nu^{-2} \omega_{\nu} \\
\geq 4^{-1} \omega_{3 n} \sum_{\nu=2 n+1}^{3 n} \nu^{-2} \geq 4^{-1} \omega_{3 n}(3 n)^{-2} n=4^{-1} 3^{-2} n^{-1} \omega_{3 n} \geq 4^{-1} 3^{-(k+2)} n^{-1} \omega_{n} .
\end{gathered}
$$

Применяя теперь известную оценку $E_{n-1}(f) \leq C_{40} n^{-1} E_{n-1}\left(f^{\prime}\right), n \in \mathbb{N}, f \in C^{1}(\mathbb{T})$ (см., например, [13, гл. III, § 1, следствие 2, неравенство (170)]), имеем

$$
4^{-1} 3^{-(k+2)} n^{-1} \omega_{n} \leq E_{n-1}\left(F_{1}\right) \leq C_{40} n^{-1} E_{n-1}\left(F_{1}^{\prime}\right)=C_{40} n^{-1} E_{n-1}\left(f_{1}\right),
$$

откуда следует оценка снизу в $n .2)$ для случая четного $k$ :

$$
E_{n-1}\left(f_{1}\right) \geq\left(4 \cdot 3^{k+2} C_{40}\right)^{-1} \omega(\pi / n), \quad n \in \mathbb{N} .
$$

При нечетном $k$ полагаем $f_{0}(x ; \omega)=f_{2}(x ; \omega)$, где (см. $[12, \S 3$, доказательство леммы 4 , формула (29)]) $f_{2}(x ; \omega)=\sum_{n=1}^{\infty} \Delta \omega_{n} \cos n x$. Очевидно, что последний ряд равномерно сходится всюду на $R$ к своей сумме $f_{2}(x ; \omega)$, так что $f_{2} \in C(\mathbb{T})$ и косинус-коэффициенты Фурье $a_{n}\left(f_{2}\right)=$ $\Delta \omega_{n}, n \in \mathbb{N}$. Поскольку $d_{n}=\sum_{\nu=n}^{\infty} a_{\nu}\left(f_{2}\right)=\sum_{\nu=n}^{\infty} \Delta \omega_{\nu}=\omega_{n}, n=\mathbb{N}$, то в силу леммы $2[12, \S 2]$ с учетом условия $\delta^{-k} \omega(\delta) \downarrow(\delta \uparrow)\left(\Rightarrow n^{k} \omega_{n} \uparrow(n \uparrow)\right)$ имеем

$$
\begin{aligned}
& \omega_{k}\left(f_{2} ; \frac{\pi}{n+2}\right) \leq \pi^{k}\left(2 k C_{38}+1\right)\left\{(n+2)^{-k} \sum_{\nu=1}^{n+1}\left|\Delta\left(\nu^{k} d_{\nu}\right)\right|+d_{n+2}\right\}+\pi^{k}\left(2^{k+1}+1\right) d_{n+3} \\
& =\pi^{k}\left(2 k C_{38}+1\right)\left\{(n+2)^{-k} \sum_{\nu=1}^{n+1}\left[(\nu+1)^{k} \omega_{\nu+1}-\nu^{k} \omega_{\nu}\right]+\omega_{n+2}\right\}+\pi^{k}\left(2^{k+1}+1\right) \omega_{n+3} \\
& \leq \pi^{k}\left(2 k C_{38}+1\right) \cdot 2 \omega_{n+2}+\pi^{k}\left(2^{k+1}+1\right) \omega_{n+2}=\pi^{k}\left(4 k C_{38}+2^{k+1}+3\right) \omega_{n+2}, \quad n \in \mathbb{N},
\end{aligned}
$$

откуда $\omega_{k}\left(f_{2} ; \pi /(n+2)\right) \leq C_{41}(k) \omega(\pi /(n+2))$, и, следовательно, $\omega_{k}\left(f_{2} ; \pi / n\right) \leq 3{ }^{k} C_{41}(k) \times$ $\omega(\pi / n), n \in \mathbb{N}$. Из последнего неравенства следует оценка сверху в $n$. 1) для случая нечетного $k$ :

$$
\omega_{k}\left(f_{2} ; \delta\right) \leq 2^{k} 3^{k} C_{41}(k) \omega(\delta), \quad \delta \in(0, \pi] .
$$

Для доказательства оценки снизу в п. 2) применяем упомянутое выше неравенство Н. К. Бари с учетом условия $n^{k} \omega_{n} \uparrow(n \uparrow)$ :

$$
E_{n-1}\left(f_{2}\right) \geq E_{n}\left(f_{2}\right) \geq 4^{-1} \sum_{\nu=2 n}^{\infty} a_{\nu}\left(f_{2}\right)=4^{-1} \sum_{\nu=2 n}^{\infty} \Delta \omega_{\nu}=4^{-1} \omega_{2 n} \geq 2^{-(k+2)} \omega_{n},
$$


откуда следует оченка снизу в $n .2)$ для случая нечетного $k$ :

$$
\omega(\pi / n) \leq 2^{k+2} E_{n-1}\left(f_{2}\right), n \in \mathbb{N} .
$$

Пользуясь леммой 1 , нетрудно установить справедливость порядковых равенств (0.3). Действительно, так как $E_{n-1}(f) \leq \rho_{n}^{(k)}(f) \leq 2^{k} C_{1}(k) \omega_{k}(f ; \pi / n), n \in \mathbb{N}$, для любого $k \in \mathbb{N}$ и каждой функции $f \in C(\mathbb{T})$, то в силу утверждений леммы 1 имеем

$$
\omega\left(\frac{\pi}{n}\right) \asymp C_{42}(k) E_{n-1}\left(f_{0}\right) \leq \rho_{n}^{(k)}\left(f_{0}\right) \leq 2^{k} C_{1}(k) \omega_{k}\left(f_{0} ; \frac{\pi}{n}\right) \asymp C_{43}(k) \omega\left(\frac{\pi}{n}\right),
$$

откуда $\rho_{n}^{(k)}\left(f_{0}\right) \asymp C_{44}(k) \omega(\pi / n), n \in \mathbb{N}$. Кроме того, поскольку $\omega_{k}\left(f_{0} ; \pi / n\right) \asymp C_{34}(k) \omega(\pi / n)$ и $E_{n-1}\left(f_{0}\right) \asymp C_{35}(k) \omega(\pi / n)$, то $E_{n-1}\left(f_{0}\right) \asymp C_{45}(k) \omega_{k}\left(f_{0} ; \pi / n\right) \asymp C_{46}(k) \omega(\pi / n), n \in \mathbb{N}$. Таким образом, все рассматриваемые величины $E_{n-1}\left(f_{0}\right), \rho_{n}^{(k)}\left(f_{0}\right)$ и $\omega_{k}\left(f_{0} ; \pi / n\right)$ имеют один и тот же порядок $\omega(\pi / n), n \in \mathbb{N}$.

\section{4. Доказательство неравенств $(0.5)-(0.7)$ и теоремы 2}

\subsection{1. Комментарий к неравенству (0.5)}

Вначале отметим, что неравенство (0.5) в несколько иной и странной формулировке фактически установлено в [14, неравенство (3)]. Странность формулировки заключается в том, что неравенство (0.5) имеет место для любой функции $f \in C(\mathbb{T})$, а не только для функций $f(x ; \varepsilon) \in C(\mathbb{T})$ (существование которых гарантируется известной теоремой С. Н. Бернштейна; см., например, [15, гл. V, $\S 5 ; 10$, гл. II, п. 2.5]), удовлетворяющих условию $E_{n}(f(\cdot ; \varepsilon))=\varepsilon_{n}$, $n \in \mathbb{Z}_{+}$, где $\varepsilon=\left\{\varepsilon_{n}\right\}_{n=0}^{\infty}-$ произвольная монотонно убывающая к нулю последовательность.

Поскольку $0 \leq E_{n}(f) \downarrow 0(n \uparrow \infty)$ для любой функции $f \in C(\mathbb{T})$, то упомянутая выше теорема С. Н. Бернштейна полностью характеризует поведение последовательности $\left\{E_{n}(f)\right\}_{n=0}^{\infty}$ в том смысле, что всякая последовательность $\varepsilon=\left\{\varepsilon_{n}\right\}_{n=0}^{\infty}$, удовлетворяющая условиям $0 \leq \varepsilon_{n} \downarrow 0$ $(n \uparrow \infty)$, является последовательностью наилучших приближений некоторой функции $f(x ; \varepsilon) \in$ $C(\mathbb{T})$ и в принципе не имеет никакого отношения к прямой теореме теории приближений. Из этой теоремы также следует, что последовательность $\left\{E_{n}(f)\right\}_{n=0}^{\infty}$ функции $f \in C(\mathbb{T})$ может стремиться к нулю с любой наперед заданной скоростью.

Кроме того, в [14] утверждается, что неравенство (0.5) в смысле порядка лучше, чем неравенство (0.1), а подтверждается этот факт указанием на функцию $f(x ; \varepsilon) \in C(\mathbb{T})$ с заданной последовательностью $E_{n}(f(\cdot ; \varepsilon))=\varepsilon_{n}=2^{-n}, n \in \mathbb{Z}_{+}$, для которой неравенство $(0.5)$ приводит к оценке $\omega_{k}(f ; \pi / n) \geq C_{47}(k) 2^{-n / 2}, n \in \mathbb{N}$, а из неравенства $(0.1)$ для этой функции вытекает лишь оценка $\omega_{k}(f ; \pi / n) \geq C_{48}(k) 2^{-n}, n \in \mathbb{N}$.

В связи с последним утверждением заметим, что поскольку для любой функции $f \in C(\mathbb{T})$ с модулем гладкости $\omega_{k}(f ; \delta) \not \equiv 0$ справедлива оценка (см., например, [16, гл. III, $\S 3$, неравенство (11)]) $\omega_{k}(f ; \delta) \geq(2 d)^{-k} \omega_{k}(f ; d) \delta^{k}, \delta \in(0, d]$, где $d-$ произвольное положительное число, то максимальный порядок убывания к нулю $\omega_{k}(f ; \delta)$ при $\delta \rightarrow 0$ есть величина $C_{49}(k) \delta^{k}$, или, что то же самое, величина $C_{50}(k) n^{-k}$ для $\omega_{k}(f ; \pi / n)$ при $n \rightarrow \infty$. Таким образом, нет никакого смысла в сравнении экспоненциальной и степенной скоростей стремления к нулю числовых последовательностей, поскольку $a^{-n}=o\left(n^{-k}\right)(n \rightarrow \infty)$ для любого $a>1$.

Далее, поскольку неравенство (0.5) является усилением неравенства (0.1), то выяснение вопроса о том, насколько такое усиление лучше, чем неравенство (0.1), следует проводить в допустимой шкале скоростей убывания к нулю модуля гладкости $\omega_{k}(f ; \pi / n)(n \rightarrow \infty)$ функции $f \in C(\mathbb{T})$. В качестве примера рассмотрим класс функций $f \in C(\mathbb{T})$, для которых $E_{n-1}(f) \asymp$ $n^{-\alpha}, n \in \mathbb{N}$, где $\alpha \in(0, k]$. Тогда в силу неравенства (0.1) имеем $\omega_{k}(f ; \pi / n) \geq C_{51}(k) n^{-\alpha}, n \in \mathbb{N}$, а в силу неравенства $(0.5)$ получаем $C_{52}(k) \omega_{k}(f ; \pi / n) \geq\left((n !)^{-1 / n}\right)^{\alpha} \geq 2^{\alpha}(n+1)^{-\alpha} \geq n^{-\alpha}$. 


\subsection{2. Доказательство неравенства (0.5)}

Мы приводим здесь несколько иное доказательство неравенства (0.5), которое, в частности, позволяет выяснить структуру постоянной величины $C_{10}(k)$. Нам понадобится известное из анализа неравенство $\left(e^{-1} n\right)^{n}<n !, n \in \mathbb{N}$, которое можно переписать в виде: $n^{n}(n !)^{-1}<e^{n}$, $n \in \mathbb{N}$. Учитывая последнее неравенство, а также неравенства $(0.1)$ и $\nu^{k} \omega_{k}(f ; \pi / \nu) \leq 2^{k} n^{k} \times$ $\omega_{k}(f ; \pi / n), 1 \leq \nu \leq n$ (см. доказательство теоремы 1 в подразд. 1.2), имеем

$$
\begin{gathered}
\prod_{\nu=1}^{n} E_{\nu-1}(f) \leq\left(C_{1}(k)\right)^{n} \prod_{\nu=1}^{n} \omega_{k}\left(f ; \frac{\pi}{\nu}\right) \leq\left(C_{1}(k)\right)^{n}\left(2^{k} n^{k} \omega_{k}\left(f ; \frac{\pi}{n}\right)\right)^{n} \prod_{\nu=1}^{n} \frac{1}{\nu^{k}} \\
=\left(C_{1}(k) 2^{k} n^{k} \omega_{k}\left(f ; \frac{\pi}{n}\right)\right)^{n} \frac{1}{(n !)^{k}}=\left(C_{1}(k) 2^{k} \omega_{k}\left(f ; \frac{\pi}{n}\right)\right)^{n}\left(\frac{n^{n}}{n !}\right)^{k}<\left(C_{1}(k) 2^{k} \omega_{k}\left(f ; \frac{\pi}{n}\right)\right)^{n} e^{k n},
\end{gathered}
$$

откуда следует неравенство $(0.5)$ с $C_{10}(k)=C_{1}(k) 2^{k} e^{k}$.

\subsection{3. Доказательство неравенства (0.6)}

Представляя указанное выше неравенство $\left(e^{-1} n\right)^{n}<n !, n \in \mathbb{N}$, в виде $1<e^{n} n^{-n} n$ !, для любого $k \in \mathbb{N}$ имеем

$$
\begin{aligned}
& \prod_{\nu=1}^{n} E_{\nu-1}(f)<e^{n k} n^{-n k}(n !)^{k} \prod_{\nu=1}^{n} E_{\nu-1}(f)=e^{n k} n^{-n k} \prod_{\nu=1}^{n} \nu^{k} E_{\nu-1}(f) \\
& \leq e^{n k} n^{-n k}\left(\max _{1 \leq \nu \leq n}\left\{\nu^{k} E_{\nu-1}(f)\right\}\right)^{n}=\left(e^{k} n^{-k} \max _{1 \leq \nu \leq n}\left\{\nu^{k} E_{\nu-1}(f)\right\}\right)^{n}
\end{aligned}
$$

откуда следует неравенство (0.6).

\subsection{4. Доказательство неравенств $(0.7)$}

Для каждого $\beta=[1, \infty)$ в силу $E_{m}(f) \downarrow(m \uparrow)$ имеем $(1 \leq \nu \leq n)$

$$
\nu^{k} E_{\nu-1}(f) \leq(\beta k)^{1 / \beta}\left(\sum_{\mu=1}^{\nu} \mu^{\beta k-1} E_{\mu-1}^{\beta}(f)\right)^{1 / \beta} \leq(\beta k)^{1 / \beta}\left(\sum_{\mu=1}^{n} \mu^{\beta k-1} E_{\mu-1}^{\beta}(f)\right)^{1 / \beta},
$$

откуда следует левое неравенство в (0.7):

$$
\rho_{n}^{(k)}(f) \equiv n^{-k} \max _{1 \leq \nu \leq n}\left\{\nu^{k} E_{\nu-1}(f)\right\} \leq(\beta k)^{1 / \beta} n^{-k}\left(\sum_{\mu=1}^{n} \mu^{\beta k-1} E_{\mu-1}^{\beta}(f)\right)^{1 / \beta} .
$$

Правое неравенство в (0.7) установлено в работе автора (см. Тр. Ин-та математики и механики УрО РАН. 2017. Т. 23, № 3. С. 144-158; разд. 1, лемма 3).

\subsection{5. Доказательство теоремы 2}

Оценка справа в (0.9) совпадает с неравенством (0.2) (см. доказательство теоремы 1). Далее, в силу неравенства (0.15) имеем

$$
\omega_{k+1}\left(f ; \frac{\pi}{n}\right) \leq C_{14}(k+1) n^{-(k+1)} \sum_{\nu=1}^{n} \nu^{k} E_{\nu-1}(f) \leq C_{14}(k+1) n^{-k} \max _{1 \leq \nu \leq n}\left\{\nu^{k} E_{\nu-1}(f)\right\},
$$

откуда и следует оценка слева в (0.9). 


\subsection{6. Об условиях на коэффициенты Фурье непрерывных функций, гарантирующих выполнение порядковых равенств (0.4) и (0.8)}

Предположим, что заданы четная функция $f \in C(\mathbb{T})$ и нечетная функция $g \in C(\mathbb{T})$ с неотрицательными коэффициентами Фурье $\left\{a_{n}(f)\right\}_{n=0}^{\infty}$ и $\left\{b_{n}(f)\right\}_{n=1}^{\infty}$. Тогда в силу известной теоремы Пэли (см., например, [17, гл. IV,$\S 2])$ ряды Фурье этих функций сходятся равномерно всюду на $\mathbb{R}$, так что имеют место равенства

$$
f(x)=\frac{a_{0}(f)}{2}+\sum_{n=1}^{\infty} a_{n}(f) \cos n x \quad \text { и } \quad g(x)=\sum_{n=1}^{\infty} b_{n}(g) \sin n x, \quad x \in \mathbb{R} .
$$

В.В. Жук $[18, \S 3$, лемма 1], пользуясь результатами о приближении функций из $C(\mathbb{T})$ линейными операторами специального вида, установил при четном $k$ справедливость (для симметрических модулей гладкости) порядкового равенства

$$
\omega_{k}\left(f ; \frac{\pi}{n}\right) \asymp C_{53}(k)\left\{n^{-k} \sum_{\nu=1}^{n} \nu^{k} a_{\nu}(f)+\sum_{\nu=n+1}^{\infty} a_{\nu}(f)\right\}, \quad n \in \mathbb{N} .
$$

Порядковое равенство (1.6) также следует из соответствующих результатов В. Э. Гейта [12]: оценка сверху имеет место в силу неравенства (4) $[12, \S 2$, лемма 1], а оценка снизу является следствием неравенств $(22)$ и $(23)[12, \S 2$, лемма 3].

Далее, учитывая очевидную оценку $E_{n-1}(f) \leq\left\|f(\cdot)-S_{n-1}(f ; \cdot)\right\| \leq \sum_{\nu=n}^{\infty} a_{\nu}(f), n \in \mathbb{N}$, а также оценку снизу в (1.6), имеем

$$
\begin{gathered}
n^{-k} \sum_{\nu=1}^{n} \nu^{k-1} E_{\nu-1}(f) \leq n^{-k} \sum_{\nu=1}^{n} \nu^{k-1} \sum_{\mu=\nu}^{n} a_{\mu}(f)+n^{-k} \sum_{\nu=1}^{n} \nu^{k-1} \sum_{\mu=n+1}^{\infty} a_{\mu}(f) \\
\leq n^{-k} \sum_{\mu=1}^{n} a_{\mu}(f) \sum_{\nu=1}^{\mu} \nu^{k-1}+\sum_{\mu=n+1}^{\infty} a_{\mu}(f) \leq n^{-k} \sum_{\mu=1}^{n} \mu^{k} a_{\mu}(f)+\sum_{\nu=n+1}^{\infty} a_{\mu}(f) \leq C_{54}(k) \omega_{k}\left(f ; \frac{\pi}{n}\right),
\end{gathered}
$$

откуда следует оценка сверху в порядковом равенстве (0.8):

$$
n^{-k} \sum_{\nu=1}^{n} \nu^{k-1} E_{\nu-1}(f) \leq C_{55}(k) \omega_{k}\left(f ; \frac{\pi}{n}\right), \quad n \in \mathbb{N}, \quad k \text { - четное. }
$$

Отметим, что оценка (1.7) другим способом ранее установлена В. В. Жуком [18, § 3, теорема 1]. Таким образом, оценка сверху в $(0.8)$ для четной функции $f$ при четном $k$ имеет место лишь при условии $a_{n}(f) \geq 0, n \in \mathbb{Z}_{+}$.

Оценки снизу в (0.8) для функции $f$ при четном $k$, а также для функции $g$ при нечетном $k$ имеют место в силу неравенства (0.15). Кроме того, оценки сверху в порядковом равенстве (0.4) для функции $f$ при нечетном $k$ и для функции $g$ при четном $k$ непосредственно следуют из неравенства $(0.2)$.

Мы не будем останавливаться на доказательстве остальных оценок, поскольку порядковые равенства (0.4) и (0.8) при некоторых дополнительных условиях на поведение коэффициентов Фурье были ранее анонсированы в кратком сообщении А.С. Белова [19, теорема 5] (там же приведена соответствующая библиография; ссылка на [18] отсутствует). В этой заметке рассматриваются классы функций $f(x)=(1 / 2) a_{0}+\sum_{n=1}^{\infty} a_{n} \cos n x$ и $g(x)=\sum_{n=1}^{\infty} b_{n} \cos n x$, где коэффициенты $a_{n} \geq 0, n \in \mathbb{Z}_{+}, b_{n} \geq 0, n \in \mathbb{N}$, удовлетворяют условиям: $\sum_{n=1}^{\infty} a_{n}<\infty$ и $n b_{n} \rightarrow 0$ при $n \rightarrow \infty$, для некоторого $\theta \in[0,+\infty)$ последовательности $n^{-\theta} a_{n} \downarrow(n \uparrow), n^{-\theta} b_{n} \downarrow(n \uparrow)$. Отметим, что условия $\sum_{n=1}^{\infty} a_{n}<\infty$ и $n b_{n} \rightarrow 0$ (где также $b_{n} \downarrow 0$ ) при $n \rightarrow \infty$, необходимы и достаточны для равномерной сходимости всюду на $\mathbb{R}$ указанных рядов к своим суммам $f(x)$ и $g(x)$ (см. [20, том I, гл. V, п. $1 ; 17$, гл. I, §30]), так что $a_{n}=a_{n}(f), n \in \mathbb{Z}_{+}, b_{n}=b_{n}(g), n \in \mathbb{N}$, и для этих функций справедливы равенства (1.5). 


\section{2. Оценка сверху $\left\|t_{n}^{(k)}(\cdot)\right\|$ посредством $\left\|\Delta_{\delta}^{k} t_{n}(\cdot)\right\|$}

\section{1. Комментарий к неравенству (0.10)}

С. Б. Стечкин называет неравенство (0.10) обобщением неравенства С. Н. Бернштейна: $\left\|t_{n}^{(k)}(\cdot)\right\| \leq n^{k}\left\|t_{n}(\cdot)\right\|$, поскольку последнее следует из $(0.10)$, если положить $\delta=\pi / n$ и воспользоваться очевидной оценкой $\left\|\Delta_{\delta}^{k} t_{n}(\cdot)\right\| \leq 2^{k}\left\|t_{n}(\cdot)\right\|$. На самом деле возможности неравенства (0.10) (и следствий из него) в плане приложений к некоторым вопросам теории приближений значительно шире (см. $[1, \S \S 4,5])$. В частности, это подтверждается справедливостью неравенств

$$
\begin{gathered}
\left\|T_{n}^{(k)}(f ; \cdot)\right\| \leq n^{k}\left(2 \sin \frac{n \delta}{2}\right)^{-k}\left\{2^{k} C_{1}(k) \omega_{k}\left(f ; \frac{\pi}{n+1}\right)+\omega_{k}(f ; \delta)\right\}, \quad 0<\delta<\frac{2 \pi}{n}, \quad n \in \mathbb{N} ; \\
\left\|T_{n}^{(k)}(f ; \cdot)\right\| \leq\left(2^{-k}+C_{1}(k)\right) n^{k} \omega_{k}\left(f ; \frac{\pi}{n}\right), \quad n \in \mathbb{N},
\end{gathered}
$$

позволяющих совершать переход от $\left\|T_{n}^{(k)}(f ; \cdot)\right\|$ к $\omega_{k}(f ; \delta)$. Эти неравенства, очевидно, не выводятся из неравенства С. Н. Бернштейна.

\section{2. Историческая справка к неравенству (0.10)}

В случае $k=1$ и $\delta=\pi / n, n \in \mathbb{N}$, утверждение, подобное неравенству (0.10), другим способом ранее установлено М. Риссом $\left[21, \S 4\right.$, теорема V , с. 361]: если $\left\|t_{n}(\cdot+\pi / n)-t_{n}(\cdot)\right\| \leq 2 M$, mо $\left\|t_{n}^{\prime}(\cdot)\right\| \leq M n$, где $M$ - некоторая постоянная величина. Впрочем, из этого утверждения вытекает неравенство (0.10) в указанном случае.

Аналог неравенства (0.10) для целых функций экспоненциального типа степени $\rho$ при $h=\pi / \rho, k \in \mathbb{N}$, получен С. М. Никольским [22], который в этой же заметке отметил, что получению указанного неравенства предшествовал следующий результат С. Б. Стечкина: $c y-$ ществует константа $C_{56}$, не зависящая от $n$, такая, что для всех тригонометрических полиномов $t_{n}(x)$ порлдка $n$ имеет место неравенство

$$
\left\|t_{n}^{\prime}(\cdot)\right\| \leq C_{56} n \sup \left\{\left\|t_{n}(\cdot+h)-t_{n}(\cdot)\right\|:|h| \leq 1 / n\right\} .
$$

\section{3. Доказательство двустороннего неравенства (0.12)}

Оценка слева в (0.12) совпадает с неравенством (0.10). Далее, поскольку $\left\|\Delta_{\delta}^{k} t_{n}(\cdot)\right\| \leq$ $\delta^{k}\left\|t_{n}^{(k)}(\cdot)\right\|$, то для доказательства оценки справа в $(0.12)$ достаточно убедиться в справедливости неравенства $(\pi / 2)^{k}((2 / n \delta) \sin (n \delta / 2))^{k} \geq 1$. Если $0<\delta \leq \pi / n$, то $0<n \delta / 2 \leq \pi / 2$, откуда в силу известного неравенства: $\sin z \geq(2 / \pi) z, z \in[0, \pi / 2]$, имеем $\sin (n \delta / 2) \geq n \delta / \pi$, и, следовательно,

$$
(\pi / 2)^{k}((2 / n \delta) \sin (n \delta / 2))^{k} \geq(\pi / 2)^{k}((2 / n \delta)(n \delta / \pi))^{k}=1 .
$$

\section{4. Доказательство теоремы 3}

Оценка сверху в порядковом равенстве $(0.14)$ следует из неравенства $\omega_{k}\left(t_{n} ; \delta\right) \leq \delta^{k}\left\|t_{n}^{(k)}(\cdot)\right\|$. Докажем оценку снизу. Если $0<\delta \leq \pi / n$, то (см. выше доказательство $(0.12)) \sin (n \delta / 2) \geq$ $n \delta / \pi$, и, следовательно, $(\sin (n \delta / 2))^{-1} \leq \pi / n \delta$. Учитывая последнюю оценку в неравенстве $(0.13)$, получаем

$$
\left\|t_{n}^{(k)}(\cdot)\right\| \leq n^{k}\left(2 \sin \left(\frac{n \delta}{2}\right)\right)^{-k} \omega_{k}\left(t_{n} ; \delta\right) \leq\left(\frac{n}{2}\right)^{k}\left(\frac{\pi}{n \delta}\right)^{k} \omega_{k}\left(t_{n} ; \delta\right)=\left(\frac{\pi}{2 \delta}\right)^{k} \omega_{k}\left(t_{n} ; \delta\right),
$$

откуда следует оценка снизу в $(0.14):(2 / \pi)^{k} \delta^{k}\left\|t_{n}^{(k)}(\cdot)\right\| \leq \omega_{k}\left(t_{n} ; \delta\right)$. 


\section{3. Обратная теорема без производных}

\section{1. Комментарии и исторические справки к неравенству (0.15) и порядковым равенствам $(0.16)-(0.17)$}

3.1.1. Неравенство (0.15), которое называется обратной теоремой (без производных) теории приближений функций $f \in C(\mathbb{T})$, как было отмечено во введении, доказано С.Б. Стечкиным в [1]. Поскольку применяемый в [1] метод доказательства этого неравенства не связан со спецификой пространства $C(\mathbb{T})$, то неравенство $(0.15)$ имеет место и для любой функции $f \in L_{p}(\mathbb{T})$ при $1 \leq p<\infty$, где $L_{p}(\mathbb{T})$ - пространство всех измеримых $2 \pi$-периодических функций с конечной $L_{p}(\mathbb{T})$-нормой $\|f\|_{p}=\left(\int_{\mathbb{T}}|f(x)|^{p} d x\right)^{1 / p}$, причем значение постоянной величины $C_{14}(k)$ в неравенстве $(0.15)$ не меняется и, следовательно, не зависит от $p$.

Ранее в докладной заметке А. Ф. Тимана и М. Ф. Тимана [23, теорема 4 , неравенство (10)] было анонсировано утверждение: если $f \in L_{p}(\mathbb{T}), 1<p<\infty$ и $h=O(1 / n)$, то $\left\|\Delta_{h}^{k} f(\cdot)\right\|_{p}=$ $O\left(n^{-k} \sum_{\nu=1}^{n} \nu^{k-1} E_{\nu}(f)_{p}\right)$, откуда сразу следует неравенство $(0.15)$ для случая функций $f \in$ $L_{p}(\mathbb{T})$ при $1<p<\infty$.

Анализ рассуждений, приведенных в [23], показал, что в неравенстве (10) из [23] постоянная величина в $O$-соотношении зависит от $k$ и $p$, причем ограничение $1<p<\infty$ является существенным, так как по сути рассуждения опирались на известное утверждение: $f \in L_{p}(\mathbb{T}) \Leftrightarrow \sup \left\{\left\|S_{n}(f ; \cdot)\right\|_{p}: n \in \mathbb{N}\right\}<\infty$, где $1<p<\infty$. Это утверждение следует (см., например, [17, гл. VIII, § 20, теоремы 1 и 2; 20, т. 1, гл. 7, п. 6, теорема (6.4)]) из неравенства М. Рисса $\left\|S_{n}(f ; \cdot)\right\|_{p} \leq C_{57}(p)\|f\|_{p}$ и вытекающего из него предельного соотношения $\left\|f(\cdot)-S_{n}(f ; \cdot)\right\|_{p} \rightarrow 0$ при $n \rightarrow \infty$.

В работе Н. К. Бари и С. Б. Стечкина $[24, \S 1$, п. $(D)]$ отмечено, что неравенство $(0.15)$ для функций $f \in L_{p}(\mathbb{T})$, где $1<p<\infty$, было впервые установлено в [23]. Позднее, в заметке [25, лемма 1, неравенство (7)] было приведено (без доказательства) неравенство, по формулировке подобное $(0.15)$, но для функции $f \in L_{p}(\mathbb{R})$ и в терминах наилучших приближений $A_{\nu}(f)_{p}$ целыми функциями экспоненциального типа степени не выше $\nu, \nu=\overline{1, n}$, где $1 \leq p \leq \infty$, и отмечено (см. первый абзац последней страницы заметки), что это неравенство (7) из [25] для периодических функций впервые было указано авторами заметки в [23, теорема 4]. Следует отметить, что о неравенстве (0.15), доказанном С.Б. Стечкиным в [1], авторы [25] забыли упомянуть, хотя ссылка на неравенство (0.1) присутствует.

Доказательство неравенства (10) [23, теорема 4] так и не было опубликовано в открытой печати вплоть до появления монографии А.Ф. Тимана [10], где приводится несколько иное в техническом исполнении, но схожее в идейном плане доказательство неравенства (0.15) (см. $\left[10\right.$, гл. 6 , теорема 6.1 .1 , неравенство (1)]) для случая $f \in L_{p}(\mathbb{T}), 1 \leq p \leq \infty$. В сноске на той же странице отмечено, что указанное там неравенство (1) получено в [23] при $p<\infty$, а распространение на случай $p=\infty$ при условии, что $f \in C(\mathbb{T})$, дано в статье С. Б. Стечкина [1]. Подобное высказывание по меньшей мере недобросовестно, поскольку в [23] неравенство (1) из [10] было лишь анонсировано (т. е. сформулировано без доказательства) при условии $1<p<\infty$, которое оказалось существенным для формулировки самой теоремы 4 [23], а С. Б. Стечкину удалось найти новый и независимый метод доказательства неравенства $(0.15)$ для функций $f \in C(\mathbb{T})$, который оказался пригодным и для случая $f \in L_{p}(\mathbb{T}), 1 \leq p<\infty$.

Позднее было обнаружено, что значительно ранее Р. Салем анонсировал [26, п. I, с. 703] (доказательство приведено в $[27$, гл. V, п. 2, с. 40-42]) следующее утверждение: nyсть $\Omega(u)-$ положительная убывающая на промежутке $(0,+\infty)$ функиия, такал, что $\Omega(u) \rightarrow 0$ при $u \rightarrow+\infty$; если для функиии $f \in C(\mathbb{T})$ существует полином $t_{n}(x)$ порлдка $n$, и для этой функици $\left\|f(\cdot)-t_{n}(\cdot)\right\| \leq \Omega(n), n \in \mathbb{N}, m o \omega(f ; \delta) \leq C_{58} \delta \int_{0}^{1 / \delta} \Omega(u) d u, \delta \in(0,1]$. В этом же сообщении $\left[26\right.$, см. сноску $\left({ }^{1}\right)$ на с. 703$]$ отмечено, что Ш. Валле-Пуссен $[8$, гл. IV, п. 39, теорема 1] доказал 
весьма близкую теорему; однако, исследуя условия существования производных функции $f$, он сделал предположение о сходимости интеграла $\int_{a}^{\infty} u^{-1} \Omega(u) d u$, где $a>1$, от которого удалось освободиться в случае $r=0$.

В случае $\Omega(u)=u^{-\alpha}$, где $u \in(0,+\infty), 0<\alpha<1$, неравенство Р. Салема приводит к правильной оценке: $\omega(f ; \delta) \leq C_{58}(1-\alpha)^{-1} \delta^{\alpha}$, а в случае $\Omega(u)=u^{-\alpha}$, где $u \in(0,+\infty)$, $\alpha \geq 1$, указанное неравенство не дает ожидаемых оценок: $\omega(f ; \delta)=O(\delta \ln (1 / \delta))$ при $\alpha=1$ и $\omega(f ; \delta)=O(\delta)$ при $\alpha>1$, где $\delta \rightarrow+0$, поскольку присутствующий в неравенстве несобственный интеграл расходится к $+\infty$. Таким образом, в случае $k=1$ Р. Салем впервые сформулировал общую обратную теорему без производных, однако полученное им утверждение не охватывает всю шкалу степенных мажорант.

Поскольку функция $\Omega(u)=u^{-\alpha}, u \in(0,+\infty), \alpha>0$, не определена в нуле, а также и не может быть доопределена в этой точке, так как $\Omega(u) \rightarrow+\infty$ при $u \rightarrow+0$, то необходимо отграничить от нуля область изменения переменной $u$, например, положить $u \in[1,+\infty)$. Очевидно, что условие $\Omega(u) \downarrow 0$ при $u \rightarrow+\infty$ выполняется на промежутке $[1,+\infty)$. В этом случае требуемое неравенство должно выглядеть следующим образом: $\omega(f ; \delta) \leq C_{59} \delta \int_{1}^{1 / \delta} \Omega(u) d u$, где $\delta \in(0, q], 0<q<1$. Тогда, полагая $\Omega(u)=u^{-\alpha}, u \in[1,+\infty), \alpha>0$, получаем, что имеют место все оценки сверху для $\omega(f ; \delta)$, приведенные в предыдущем абзаце.

На основании изложенного и руководствуясь принципом исторической справедливости, автор считает правильным называть неравенство (0.15) неравенством Салема - Стечкина.

3.1.2. С. Н. Бернштейн впервые поставил задачу об обратных теоремах теории приближений и при $k=1$ установил [28, гл. II, § 25] справедливость импликации $E_{n-1}(f)=O\left(n^{-\alpha}\right)$, $n \in \mathbb{N} \Rightarrow \omega(f ; \delta)=O\left(\delta^{\alpha-\varepsilon}\right), \delta \in(0, \pi]$, где $0<\alpha \leq 1, \alpha-\varepsilon=\alpha /(1+\alpha) \Leftrightarrow \varepsilon=\alpha^{2} /(1+\alpha) \in(0, \alpha)$. В этой же работе [28, гл. II, § 24] им были введены модули непрерывности старших порядков $\omega_{k}(f ; \delta), k=1,2,3, \ldots$, и доказано следующее утверждение (см. там же $\left.\S 25\right): е с л и$ $E_{n-1}(f)=O\left(n^{-\alpha}\right), n \in \mathbb{N}$, mo $\omega_{k}(f ; \delta)=O\left(\delta^{\alpha-\varepsilon}\right), \delta \in(0, \pi]$, где $\alpha-\varepsilon=\alpha k /(k+\alpha) \Leftrightarrow \varepsilon=$ $\alpha^{2} /(k+\alpha) \in(0, \alpha)$.

Ш. Валле-Пуссен [8, гл. IV, п. 41, теорема III и п. 44, теорема V] уточнил сформулированный выше результат С. Н. Бернштейна в случае $k=1$, а именно: если $0<\alpha<1 u E_{n-1}(f)=$ $O\left(n^{-\alpha}\right), n \in \mathbb{N}$, то $\omega(f ; \delta)=O\left(\delta^{\alpha}\right), \delta \in(0, \pi]$, а при $\alpha=1$ условие $E_{n-1}(f)=O\left(n^{-1}\right), n \in \mathbb{N}$, влечет $\omega(f ; \delta)=O(\delta \ln (\pi e / \delta)), \delta \in(0, \pi]$ (последнее заключение было также отмечено в [7, п. 1 , первый абзац на с. 52]). Приведенные утверждения являются следствиями более общей теоремы Ш. Валле-Пуссена [8, гл. IV, п. 39, теорема I].

Э. Квад [29, теорема 1] установил, что упомянутая выше общая теорема Ш. Валле-Пуссена имеет место и в случае функций $f \in L_{p}(\mathbb{T})$, где $1 \leq p<\infty$, причем формулировки соответствующих основных неравенств (см. [8, гл. IV, п. 39, теорема I, неравенство (2)] и [29, теорема 1, неравенство (1)]) остаются без изменения. Кроме того, в этой же работе [29, теорема 2] доказано, что приведенные в предыдущем абзаце уточнения оценки $\omega(f ; \delta)$ остаются в силе также и в рассматриваемом случае: если $f \in L_{p}(\mathbb{T}), 1 \leq p<\infty, 0<\alpha \leq 1 u$ $\left\|f(\cdot)-t_{n}(\cdot)\right\|_{p}=O\left(n^{-\alpha}\right), \quad n \in \mathbb{N}$, mo $\omega(f ; \delta)_{p}=O\left(\delta^{\alpha}\right)$ npu $\alpha<1 u \omega(f ; \delta)_{p}=O(\delta \ln (\pi e / \delta))$ при $\alpha=1, \delta \in(0, \pi]$.

А. Зигмунд [7] впервые обратил внимание на то, что привлечение модулей непрерывности второго порядка $\omega_{2}(f ; \delta)$ позволяет избежать появления логарифмического множителя при оценке $\omega(f ; \delta)$ для функций $f \in C(\mathbb{T})$ с $E_{n-1}(f)=O\left(n^{-1}\right), n \in \mathbb{N}$, а именно, им установлена (см. [7, п. 1 , теорема 8, с. 52 ; доказательство приведено в п. 5, с. 61-62]) справедливость следующего утверждения: если $f \in C(\mathbb{T})$, то $E_{n-1}(f)=O\left(n^{-1}\right), n \in \mathbb{N} \Leftrightarrow \omega_{2}(f ; \delta)=O(\delta), \delta \in(0, \pi]$.

Последний результат в силу соотношения $\omega(f ; \delta)=O\left(\delta^{\alpha}\right) \Leftrightarrow \omega_{2}(f ; \delta)=O\left(\delta^{\alpha}\right)$ при $0<\alpha<1$, установленного там же (см. [7, п. 5, лемма 4, с. 63]), следует также из общего утверждения в случае $\alpha=1$ : если $f \in C(\mathbb{T}), 0<\alpha \leq 1$, mo $E_{n-1}(f)=O\left(n^{-\alpha}\right), n \in \mathbb{N} \Leftrightarrow$ $\omega_{2}(f ; \delta)=O\left(\delta^{\alpha}\right), \delta \in(0 ; \pi]$. 
В этой же работе показано [7, п. 1, теорема 9, с. 52, доказательство приведено в п. 5, с. 62 , а также см. п. 5 , замечание $(i i)$, с. 64$]$, что аналогичный результат имеет место и для модулей непрерывности высших порядков, а именно: если $f \in C(\mathbb{T}), k \in \mathbb{N}, m_{0} E_{n-1}(f)=O\left(n^{-(k+1)}\right)$, $n \in \mathbb{N} \Leftrightarrow \omega_{k+2}(f ; \delta)=O\left(\delta^{k+1}\right), \delta \in(0, \pi], u E_{n-1}(f)=O\left(n^{-(k+\alpha)}\right), \quad n \in \mathbb{N} \Leftrightarrow \omega_{k+1}(f ; \delta)=$ $O\left(\delta^{k+\alpha}\right), \delta \in(0, \pi]$, где $0<\alpha<1$.

3.1.3. Порядковое равенство (0.16), хотя и не выписано, фактически следует из соответствующих неравенств, приведенных в [1]: по поводу оценки сверху см. $\S 5$, доказательство леммы 9 и $\S 6$, доказательство леммы 11, а оценка снизу имеет место в силу неравенства (0.1) и неравенства (см. там же $\S 4$, теорема 3 , неравенство (4.5)):

$$
\left\|T_{n}^{(k)}(f)\right\| \leq(n+1)^{k}\left(C_{1}(k)+1\right) \omega_{k}(f ; \pi /(n+1)), n \in \mathbb{Z}_{+} .
$$

3.1.4. Порядковое равенство (0.17) установлено В. В. Жуком [13, гл. VII, § 8, теорема 2]:

$$
C_{60}(k) \omega_{k}\left(f ; \frac{\pi}{n}\right) \leq\left\|f-T_{n}(f)+n^{-k} T_{n}^{(k)}(f)\right\| \leq C_{61}(k) \omega_{k}\left(f ; \frac{\pi}{n}\right), \quad n \in \mathbb{N}
$$

где $C_{60}(k)=\left(2^{k}+2 \pi^{k}\right)^{-1}, C_{61}(k)=2^{-k}+2 C_{1}(k), C_{1}(k)$ - постоянная величина в $(0.1)$.

\section{2. Доказательство теоремы 4}

Достаточность: пусть имеет место неравенство (0.19). В силу известных свойств модулей гладкости имеем

$$
\begin{gathered}
\omega_{k}\left(f ; \frac{\pi}{n}\right) \leq \omega_{k}\left(f-T_{n}(f) ; \frac{\pi}{n}\right)+\omega_{k}\left(T_{n}(f) ; \frac{\pi}{n}\right) \leq 2^{k}\left\|f-T_{n}(f)\right\|+\pi^{k} n^{-k}\left\|T_{n}^{(k)}(f)\right\| \\
=2^{k} E_{n}(f)+\pi^{k} n^{-k}\left\|T_{n}^{(k)}(f)\right\| \leq k 2^{k} n^{-k} \sum_{\nu=1}^{n} \nu^{k-1} E_{\nu-1}(f)+\pi^{k} n^{-k} C_{19}(k) \sum_{\nu=1}^{n} \nu^{k-1} E_{\nu-1}(f) \\
=\left(k 2^{k}+\pi^{k} C_{19}(k)\right) n^{-k} \sum_{\nu=1}^{n} \nu^{k-1} E_{\nu-1}(f), \quad n \in \mathbb{N},
\end{gathered}
$$

откуда следует неравенство (0.15) с $C_{14}(k)=k 2^{k}+\pi^{k} C_{19}(k)$.

Необходимостъ: пусть имеет место неравенство (0.15). Полагая в неравенстве $(0.10) t_{n}(\cdot)=$ $T_{n}(f ; \cdot), \delta=\pi / n$ и учитывая неравенство $(0.1)$, получаем

$$
\begin{gathered}
\left\|T_{n}^{(k)}(f ; \cdot)\right\| \leq 2^{-k} n^{k}\left\|\Delta_{\pi / n}^{k} T_{n}(f ; \cdot)\right\| \leq 2^{-k} n^{k}\left\{\left\|\Delta_{\pi / n}^{k}\left[T_{n}(f ; \cdot)-f(\cdot)\right]\right\|+\left\|\Delta_{\pi / n}^{k} f(\cdot)\right\|\right\} \\
\leq 2^{-k} n^{k}\left\{2^{k}\left\|T_{n}(f ; \cdot)-f(\cdot)\right\|+\left\|\Delta_{\pi / n}^{k} f(\cdot)\right\|\right\} \leq n^{k}\left\{E_{n}(f)+2^{-k} \omega_{k}\left(f ; \frac{\pi}{n}\right)\right\} \\
\leq n^{k}\left\{C_{1}(k) \omega_{k}\left(f ; \frac{\pi}{n+1}\right)+2^{-k} \omega_{k}\left(f ; \frac{\pi}{n}\right)\right\} \leq\left(C_{1}(k)+2^{-k}\right) n^{k} \omega_{k}\left(f ; \frac{\pi}{n}\right) \\
\leq\left(C_{1}(k)+2^{-k}\right) C_{14}(k) \sum_{\nu=1}^{n} \nu^{k-1} E_{\nu-1}(f), \quad n \in \mathbb{N},
\end{gathered}
$$

откуда следует неравенство $(0.19)$ с $C_{19}(k)=C_{14}(k)\left(2^{-k}+C_{1}(k)\right)$. 


\section{4. Обратная теорема с производными}

\section{1. Историческая справка}

Первая часть утверждения

$$
E(f ; r) \equiv \sum_{n=1}^{\infty} n^{r-1} E_{n-1}(f)<\infty \Rightarrow f \in C^{r}(\mathbb{T})
$$

принадлежит С.Н. Бернштейну (см. доказательство теоремы 10 в [1], а также приведенное ниже доказательство теоремы 5 в части "необходимость"). Неравенство (0.20) впервые установлено С. Б. Стечкиным $[1, \S 5$, следствие 10.1$]$. В работе [1] также отмечается (см. сноску на странице, где приведена формулировка теоремы 11), что А. Ф. Тиман получил независимо от С. Б. Стечкина доказательство (0.21) (без ссылки на какой-либо источник), а в работе $[24, \S 1$, последний абзац п. (Д)] последнее утверждение подкрепляется ссылкой на автореферат докторской диссертации А. Ф. Тимана (Днепропетровск, 1951). До появления монографии А.Ф. Тимана [10] доказательство неравенства (0.21) нигде ранее не было опубликовано, а в [10, гл. VI, теорема 6.1.3] приводится ссылка на саму диссертацию А.Ф. Тимана (Харьков, 1951).

Первая попытка формулировки обратной теоремы в общем виде (как без производных, так и с производными) была предпринята Ш. Валле-Пуссеном [8, гл. IV, п. 39, теорема 1 , неравенство (2)] (см. также разд. 3 нашей статьи, подразделы 3.1.1 и 3.1.2). Для дальнейшего изложения нам понадобится формулировка этой теоремы в том виде, как это было сделано Э. Квадом [29, теорема 1, с. 532], поскольку она нуждается в необходимых комментариях.

Пусть задана функиия $\Omega(u) \not \equiv$, которая удовлетворяет условиям: $\Omega(u) \geq 0, u \in(0,+\infty)$, $u$, монотонно убывая на $\left[u_{0},+\infty\right)$, стремится к нулю при $u \rightarrow+\infty$, где $u_{0}-$ некоторая фиксированная точка из промежутка $(0,+\infty)$, причем интеграл $\int_{u_{0}}^{+\infty} u^{-1} \Omega(u) d u$ сходится. Если $2 \pi$-периодическая функиия $f$ для каждого $n \in \mathbb{N}$ допускает приближение тригонометрическим полиномом порядка не выше $n$, так что $\left\|f(\cdot)-t_{n}(\cdot)\right\| \leq n^{-r} \Omega(n), r \in \mathbb{Z}_{+}$, то $f$ имеет производную $f^{(r)} \in C(\mathbb{T})\left(f^{(0)} \equiv f\right.$ при $\left.r=0\right)$, для которой выполняется неравенство

$$
\omega\left(f^{(r)} ; \delta\right) \leq C_{62}(r, a)\left\{\delta \int_{a}^{a / \delta} \Omega(u) d u+\int_{1 / \delta}^{+\infty} u^{-1} \Omega(u) d u\right\},
$$

где а - константа, которая может зависеть от $f$, но не от $\delta$.

Кроме того, в этой же работе [8, гл. IV, п. 40, теорема II] доказано, что если в условиях общей теоремы функиия $u^{-\alpha} \Omega(u)$ при некотором $\alpha<1$ не является убывающей на промежутке $[a,+\infty)$, где $a>1$, то справедливо неравенство

$$
\omega\left(f^{(r)} ; \delta\right) \leq C_{62}(r, a) C_{63}(a, \alpha) \int_{1 / \delta}^{+\infty} u^{-1} \Omega(u) d u .
$$

Очевидно, что можно положить $u_{0}=1$, и тогда указанный интеграл сходится при любом $a>1$, а условие $\Omega(u) \downarrow 0(u \uparrow+\infty)$ выполняется на любом промежутке $[a,+\infty)$.

Наличие константы $a$ в (4.1) связано лишь с тем, что она участвует в роли технического параметра при доказательстве этого неравенства и никоим образом не влияет на скорость убывания к нулю правой части (4.1) при $\delta \rightarrow+0$. Ш. Валле-Пуссен [8, гл. IV, п. 39, доказательство теоремы 1] использовал произвольное число $a>1$ и получил значение $C_{62}(r, a)=$ $2 a^{r+2}(a-1)^{-1}$, а Э. Квад [29, доказательство теоремы 1$]-$ число $a \geq 2$ и получил значение $C_{64}(r, a)=2(2 a)^{r+2}(a-1)^{-1}=2^{r+2} C_{62}(r, a)$. 
В случае $r=0$ непрерывность функции $f$ обеспечивается условием $\left\|f(\cdot)-t_{n}(\cdot)\right\| \leq \Omega(n) \downarrow 0$ $(n \uparrow \infty)$, а в случае $r \geq 1$ принадлежность $f \in C^{r}(\mathbb{T})$ гарантируется сходимостью интеграла $\int_{1}^{+\infty} u^{-1} \Omega(u) d u$. Следовательно, в случае $r=0$ нет необходимости в привлечении условия сходимости указанного интеграла, а также в присутствии второго слагаемого в правой части (4.1) (последнее наблюдение было позднее отмечено в заметке Р. Салема [26]; см. выше разд. 3, подразд. 3.1.1). Однако, несмотря на это, неравенство (4.1) позволяет получить ожидаемые правильные оценки для $\omega\left(f^{(r)} ; \delta\right)$ как в случае $r=0$, так и в случае $r \geq 1$.

Полагая $\Omega(u)=u^{-\alpha}, 0<\alpha<1$, в неравенстве (4.2) и $\Omega(u)=u^{-1}-$ в неравенстве (4.1), Ш. Валле-Пуссен получает следующее утверждение $[8$, гл. IV, п. 41, теорема III и п. 44, теорема V]: если $\left\|f(\cdot)-t_{n}(\cdot)\right\|=O\left(n^{-(r+\alpha)}\right)$, где $n \in \mathbb{N}, r \in \mathbb{Z}_{+}, 0<\alpha \leq 1$, mo $f \in C^{r}(\mathbb{T})$, $\omega\left(f^{(r)} ; \delta\right)=O\left(\delta^{\alpha}\right)$ nрu $\alpha<1 u \omega\left(f^{(r)} ; \delta\right)=O(\delta \ln (e / \delta))$ npu $\alpha=1$.

Следует также отметить один результат А.Зигмунда [7, п. 1, теорема 9, с. 52; доказательство приведено в п. 5, с. 62], позволяющий избежать появления логарифмического множителя при $\alpha=1$ : если $f \in C(\mathbb{T}), r \in \mathbb{N}$, mо $E_{n}(f)=O\left(n^{-(r+1)}\right), n \in \mathbb{N} \Leftrightarrow f \in C^{r}(\mathbb{T}) u$ $\omega_{2}\left(f^{(r)} ; \delta\right)=O(\delta), \delta \in(0, \pi]$.

\section{2. Основная вспомогательная лемма}

В следующей лемме собраны некоторые оценки для $E_{n}(f)$ и $T_{n}(f ; \cdot)$, используемые при доказательстве приведенных ниже утверждений.

Лемма 2. Пусть $f \in C(\mathbb{T}), T_{n}(f ; x) \equiv T_{n}(f), r \in \mathbb{N} ;$ тогда

1) $E_{n}(f) \leq\|f\|,\left\|T_{n}(f)\right\| \leq 2\|f\|, n \in \mathbb{Z}_{+}$;

2) $E_{\nu-1}\left(T_{n}(f)\right) \leq 2 E_{\nu-1}(f), 1 \leq \nu \leq n, n \in \mathbb{N}, \quad E_{\nu-1}\left(T_{n}(f)\right)=0, \nu \geq n+1, n \in \mathbb{Z}_{+}$;

3) $E_{\nu-1}\left(f-T_{n}(f)\right)=E_{n}(f), 1 \leq \nu \leq n, n \in \mathbb{N}, \quad E_{\nu-1}\left(f-T_{n}(f)\right)=E_{\nu-1}(f), \quad \nu \geq n+1$, $n \in \mathbb{Z}_{+}$

4) $E_{n}\left(f^{(r)}\right) \leq\left\|f^{(r)}-T_{n}^{(r)}(f)\right\| \leq C_{65}(r) E_{n}\left(f^{(r)}\right), n \in \mathbb{N}, f \in C^{r}(\mathbb{T})$;

5) $E_{n-1}\left(T_{n}^{(r)}(f)\right) \leq 2 n^{r} E_{n-1}(f), n \in \mathbb{N}$.

Д о к а з а т е л ь с т в о. 1) Оценка слева известна (см., например, [13, гл. II, § 7, лемма 2]). Оценка справа является следствием оценки слева:

$$
\left\|T_{n}(f)\right\| \leq\left\|T_{n}(f)-f\right\|+\|f\|=E_{n}(f)+\|f\| \leq 2\|f\| .
$$

2) В силу известных свойств последовательности $\left\{E_{n}(f)\right\}_{n=0}^{\infty}$ имеем $(1 \leq \nu \leq n, n \in \mathbb{N})$ :

$$
\begin{gathered}
E_{\nu-1}\left(T_{n}(f)\right) \leq E_{\nu-1}\left(T_{n}(f)-f\right)+E_{\nu-1}(f) \\
\leq\left\|T_{n}(f)-f\right\|+E_{\nu-1}(f)=E_{n}(f)+E_{\nu-1}(f) \leq 2 E_{\nu-1}(f),
\end{gathered}
$$

откуда следует оценка слева. Оценка справа очевидна.

3) Оценка слева:

$$
\begin{gathered}
E_{\nu-1}\left(f-T_{n}(f)\right) \leq\left\|f-T_{n}(f)\right\|=E_{n}(f) \leq E_{n}\left(f-T_{n}(f)\right)+E_{n}\left(T_{n}(f)\right) \\
=E_{n}\left(f-T_{n}(f)\right) \leq E_{\nu-1}\left(f-T_{n}(f)\right), \quad 1 \leq \nu \leq n, \quad n \in \mathbb{N} .
\end{gathered}
$$

Оценка справа:

$$
E_{\nu-1}\left(f-T_{n}(f)\right) \leq E_{\nu-1}(f)+E_{\nu-1}\left(T_{n}(f)\right)=E_{\nu-1}(f)
$$




$$
\leq E_{\nu-1}\left(f-T_{n}(f)\right)+E_{\nu-1}\left(T_{n}(f)\right)=E_{\nu-1}\left(f-T_{n}(f)\right), \quad \nu \geq n+1, \quad n \in \mathbb{Z}_{+} .
$$

4) Оценка слева очевидна. Доказательство оценки справа приведено, например, в [13, гл. III, § 6, следствие 2, неравенство (217)].

5) Применяя неравенство С. Н. Бернштейна $\left\|t_{n}^{(r)}(\cdot)\right\| \leq n^{r}\left\|t_{n}(\cdot)\right\|$ и учитывая свойство монотонности $E_{n}(f) \downarrow(n \uparrow)$, получаем

$$
\begin{gathered}
E_{n-1}\left(T_{n}^{(r)}(f)\right) \leq\left\|T_{n}^{(r)}(f)-T_{n-1}^{(r)}(f)\right\|=\left\|\left\{T_{n}(f)-T_{n-1}(f)\right\}^{(r)}\right\| \leq n^{r}\left\|T_{n}(f)-T_{n-1}(f)\right\| \\
\leq n^{r}\left\{\left\|T_{n}(f)-f\right\|+\left\|f-T_{n-1}(f)\right\|\right\}=n^{r}\left\{E_{n}(f)+E_{n-1}(f)\right\} \leq 2 n^{r} E_{n-1}(f) .
\end{gathered}
$$

Следует отметить, что применение неравенства $(0.20)$ к полиному $T_{n}(f)$ также приводит к оценке в п. 5$)$, но с константой $2 C_{20}(r)$, однако при этом получается более тонкая промежуточная оценка

$$
E_{n-1}\left(T_{n}^{(r)}(f)\right) \leq C_{20}(r) n^{r} E_{n-1}\left(T_{n}(f)\right), n \in \mathbb{N} .
$$

Действительно, учитывая оценки в п. 2) леммы 2, имеем

$$
\begin{gathered}
E_{n-1}\left(T_{n}^{(r)}(f)\right) \leq C_{20}(r)\left\{n^{r} E_{n-1}\left(T_{n}(f)\right)+\sum_{\nu=n+1}^{\infty} \nu^{r-1} E_{\nu-1}\left(T_{n}(f)\right)\right\} \\
=C_{20}(r) n^{r} E_{n-1}\left(T_{n}(f)\right) \leq 2 C_{20}(r) n^{r} E_{n-1}(f), \quad n \in \mathbb{N} .
\end{gathered}
$$

\section{3. Доказательство теоремы 5}

Достаточность: если имеет место неравенство $(0.22)$, то, полагая в нем $f(x)=T_{n}(f ; x)$ и учитывая оценки в п. 2) леммы 2, получаем

$$
\begin{gathered}
\left\|T_{n}^{(r)}(f)\right\| \leq C_{22}(r) E\left(T_{n}(f) ; r\right)=C_{22}(r) \sum_{\nu=1}^{\infty} \nu^{r-1} E_{\nu-1}\left(T_{n}(f)\right) \\
=C_{22}(r)\left\{\sum_{\nu=1}^{n} \nu^{r-1} E_{\nu-1}\left(T_{n}(f)\right)+\sum_{\nu=n+1}^{\infty} \nu^{r-1} E_{\nu-1}\left(T_{n}(f)\right)\right\} \leq 2 C_{22}(r) \sum_{\nu=1}^{n} \nu^{r-1} E_{\nu-1}(f) .
\end{gathered}
$$

Необходимостъ: пусть имеет место неравенство (0.23). В статье С. Б. Стечкина $[1, \S 5$, доказательство теоремы 10] указывается, что С. Н. Бернштейном [28] доказана теорема

$$
E(f ; r)<\infty \Rightarrow f \in C^{r}(\mathbb{T}),
$$

причем в ходе доказательства этой теоремы было установлено (как и при доказательстве самой теоремы 10 в [1]), что $\left\|T_{n}^{(r)}(f)-f^{(r)}\right\| \rightarrow 0$ при $n \rightarrow \infty$, откуда следует предельное соотношение $\left\|T_{n}^{(r)}(f)\right\| \rightarrow\left\|f^{(r)}\right\|(n \rightarrow \infty)$, поскольку $\left\|T_{n}^{(r)}(f)\right\|-\left\|f^{(r)}\right\| \mid \leq\left\|T_{n}^{(r)}(f)-f^{(r)}\right\|, n \in \mathbb{N}$. Отсюда, переходя к пределу при $n \rightarrow \infty$ в неравенстве (0.23), получаем

$$
\left\|f^{(r)}\right\|=\lim _{n \rightarrow \infty}\left\|T_{n}^{(r)}(f)\right\| \leq C_{19}(r) E(f ; r) .
$$

Отметим, что последнюю оценку можно получить, не привлекая предельный переход: поскольку $E(f ; r)<\infty \Rightarrow f \in C^{r}(\mathbb{T})$ и $\left\|T_{n}^{(r)}(f)-f^{(r)}\right\| \rightarrow 0$ при $n \rightarrow \infty$, то из очевидного неравенства $\left\|f^{(r)}\right\| \leq\left\|f^{(r)}-T_{n}^{(r)}(f)\right\|+\left\|T_{n}^{(r)}(f)\right\|$ следует оценка

$$
\left\|f^{(r)}\right\| \leq \sup \left\{\left\|T_{n}^{(r)}(f)\right\|: n \in \mathbb{N}\right\} \leq C_{19}(r) E(f ; r) .
$$




\section{4. Доказательство теоремы 6}

Достаточностъ: если имеет место неравенство (0.22), то, применяя его к функции $g_{n}(x)=$ $f(x)-T_{n}(f ; x), n \in \mathbb{N}$, и учитывая оценки в п. 3$)$ леммы 2 , получаем

$$
\begin{gathered}
E_{n}\left(f^{(r)}\right) \leq\left\|f^{(r)}-T_{n}^{(r)}(f)\right\|=\left\|g_{n}^{(r)}\right\| \leq C_{22}(r) E\left(g_{n} ; r\right)=C_{22}(r) \sum_{\nu=1}^{\infty} \nu^{r-1} E_{\nu-1}\left(g_{n}\right) \\
=C_{22}(r)\left\{\sum_{\nu=1}^{n+1} \nu^{r-1} E_{\nu-1}\left(g_{n}\right)+\sum_{\nu=n+2}^{\infty} \nu^{r-1} E_{\nu-1}\left(g_{n}\right)\right\} \leq C_{22}(r)\left\{(n+1)^{r} E_{n}(f)+\sum_{\nu=n+2}^{\infty} \nu^{r-1} E_{\nu-1}(f)\right\} .
\end{gathered}
$$

Оценка сверху для $E_{0}\left(f^{(r)}\right)$ непосредственно следует из неравенства $(0.22)$ :

$$
E_{0}\left(f^{(r)}\right) \leq\left\|f^{(r)}\right\| \leq C_{22}(r) E(f ; r)=C_{22}(r)\left\{E_{0}(f)+\sum_{\nu=2}^{\infty} \nu^{r-1} E_{\nu-1}(f)\right\} .
$$

Необходимость: если имеет место неравенство (0.20), то в силу неравенства справа в п. 4) леммы 2 и неравенства (0.23) имеем при любом $n \in \mathbb{N}$

$$
\begin{gathered}
\left\|f^{(r)}\right\| \leq\left\|f^{(r)}-T_{n}^{(r)}(f)\right\|+\left\|T_{n}^{(r)}(f)\right\| \leq C_{65}(r) E_{n}\left(f^{(r)}\right)+\left\|T_{n}^{(r)}(f)\right\| \\
\leq C_{65}(r) C_{20}(r)\left\{(n+1)^{r} E_{n}(f)+\sum_{\nu=n+2}^{\infty} \nu^{r-1} E_{\nu-1}(f)\right\}+C_{19}(r) \sum_{\nu=1}^{n} \nu^{r-1} E_{\nu-1}(f) \\
\leq\left(C_{65}(r) C_{20}(r) r+C_{19}(r)\right) \sum_{\nu=1}^{\infty} \nu^{r-1} E_{\nu-1}(f)=\left(r C_{65}(r) C_{20}(r)+C_{19}(r)\right) E(f ; r) .
\end{gathered}
$$

\section{5. Доказательство теоремы 7}

Достаточность: пусть имеет место неравенство (0.21). Применяя это неравенство к функции $g_{n}(x)=f(x)-T_{n}(f ; x), n \in \mathbb{N}$, и учитывая оценки в п. 3) леммы 2 , имеем

$$
\begin{gathered}
\omega_{k}\left(g_{n}^{(r)} ; \frac{\pi}{n}\right) \leq C_{21}(k, r)\left\{n^{-k} \sum_{\nu=1}^{n} \nu^{k+r-1} E_{\nu-1}\left(g_{n}\right)+\sum_{\nu=n+1}^{\infty} \nu^{r-1} E_{\nu-1}\left(g_{n}\right)\right\} \\
=C_{21}(k, r)\left\{E_{n}(f) n^{-k} \sum_{\nu=1}^{n} \nu^{k+r-1}+\sum_{\nu=n+1}^{\infty} \nu^{r-1} E_{\nu-1}(f)\right\} \\
\leq C_{21}(k, r)\left\{n^{r} E_{n}(f)+\sum_{\nu=n+1}^{\infty} \nu^{r-1} E_{\nu-1}(f)\right\} .
\end{gathered}
$$

Учитывая последнюю оценку, неравенство (0.1) и оценку в п. 5) леммы 2, получаем

$$
\begin{gathered}
E_{n-1}\left(f^{(r)}\right) \leq E_{n-1}\left(f^{(r)}-T_{n}^{(r)}(f)\right)+E_{n-1}\left(T_{n}^{(r)}(f)\right)=E_{n-1}\left(g_{n}^{(r)}\right)+E_{n-1}\left(T_{n}^{(r)}(f)\right) \\
\leq C_{1}(k) \omega_{k}\left(g_{n}^{(r)} ; \frac{\pi}{n}\right)+2 n^{r} E_{n-1}(f) \leq C_{1}(k) C_{21}(k, r)\left\{n^{r} E_{n}(f)+\sum_{\nu=n+1}^{\infty} \nu^{r-1} E_{\nu-1}(f)\right\}+2 n^{r} E_{n-1}(f) \\
\leq\left(C_{1}(k) C_{21}(k, r)+2\right)\left\{n^{r} E_{n-1}(f)+\sum_{\nu=n+1}^{\infty} \nu^{r-1} E_{\nu-1}(f)\right\} .
\end{gathered}
$$


Необходимость: пусть имеет место неравенство (0.20). Тогда, учитывая также оценку справа в п. 4) леммы 2 и неравенство (0.19) для значения $r+k$ вместо $k$, имеем

$$
\begin{gathered}
\omega_{k}\left(f^{(r)} ; \frac{\pi}{n}\right) \leq \omega_{k}\left(f^{(r)}-T_{n}^{(r)}(f) ; \frac{\pi}{n}\right)+\omega_{k}\left(T_{n}^{(r)}(f) ; \frac{\pi}{n}\right) \leq 2^{k}\left\|f^{(r)}-T_{n}^{(r)}(f)\right\| \\
+\pi^{k} n^{-k}\left\|T_{n}^{(r+k)}(f)\right\| \leq 2^{k} C_{65}(r) E_{n}\left(f^{(r)}\right)+\pi^{k} n^{-k}\left\|T_{n}^{(r+k)}(f)\right\| \\
\leq 2^{k} C_{65}(r) C_{20}(r)\left\{n^{r} E_{n-1}(f)+\sum_{\nu=n+1}^{\infty} \nu^{r-1} E_{\nu-1}(f)\right\}+\pi^{k} C_{19}(r+k) n^{-k} \sum_{\nu=1}^{n} \nu^{r+k-1} E_{\nu-1}(f) \\
\leq\left((r+k) 2^{k} C_{65}(r) C_{20}(r)+\pi^{k} C_{19}(r+k)\right)\left\{n^{-k} \sum_{\nu=1}^{n} \nu^{r+k-1} E_{\nu-1}(f)+\sum_{\nu=n+1}^{\infty} \nu^{r-1} E_{\nu-1}(f)\right\} .
\end{gathered}
$$

3 а м е ч а н и е 2. Выполнение неравенств (0.20) и (0.19) со значением $r+k$ вместо $k$, используемых для доказательства неравенства (0.21) (см. доказательство теоремы 7 в части "необходимость"), не только достаточно, но и необходимо для справедливости неравенства (0.21). Необходимость выполнения неравенства (0.20) установлена при доказательстве теоремы 7 в части “достаточность". Докажем необходимость выполнения неравенства (0.19) со значением $r+k$ вместо $k$. В силу неравенства (0.10) для значения $\delta=\pi / n$ и оценок в п. 2$)$ леммы 2 имеем

$$
\begin{gathered}
\left\|T_{n}^{(r+k)}(f)\right\|=\left\|\left(T_{n}^{(r)}(f)\right)^{(k)}\right\| \leq 2^{-k} n^{k}\left\|\Delta_{\pi / n}^{k} T_{n}^{(r)}(f ; \cdot)\right\| \leq 2^{-k} n^{k} \omega_{k}\left(T_{n}^{(r)}(f) ; \frac{\pi}{n}\right) \\
\leq 2^{-k} n^{k} C_{21}(k, r)\left\{n^{-k} \sum_{\nu=1}^{n} \nu^{k+r-1} E_{\nu-1}\left(T_{n}(f)\right)+\sum_{\nu=n+1}^{\infty} \nu^{r-1} E_{\nu-1}\left(T_{n}(f)\right)\right\} \\
\leq 2^{-k} n^{k} C_{21}(k, r) 2 n^{-k} \sum_{\nu=1}^{n} \nu^{k+r-1} E_{\nu-1}(f)=2^{-k+1} C_{21}(k, r) \sum_{\nu=1}^{n} \nu^{r+k-1} E_{\nu-1}(f) .
\end{gathered}
$$

\section{6. Доказательство теоремы 8}

Достаточность: пусть имеет место неравенство (0.22). Тогда в силу теоремы 6 в части "достаточность" справедливо неравенство (0.20). Отсюда так же, как и при доказательстве теоремы 7 в части "необходимость", получаем

$$
\begin{gathered}
\omega_{k}\left(f^{(r)} ; \frac{\pi}{n}\right) \leq 2^{k} C_{65}(r) E_{n-1}\left(f^{(r)}\right)+\pi^{k} n^{-k}\left\|T_{n}^{(r+k)}(f)\right\| \\
\leq\left((r+k) 2^{k} C_{65}(r) C_{20}(r)+\pi^{k} C_{19}(r+k)\right)\left\{n^{-k} \sum_{\nu=1}^{n} \nu^{k+r-1} E_{\nu-1}(f)+\sum_{\nu=n+1}^{\infty} \nu^{r-1} E_{\nu-1}(f)\right\} .
\end{gathered}
$$

Необходимость: пусть теперь выполняется (0.21). Тогда, учитывая оценку справа в п. 4) леммы 2, а также неравенства (0.1) и (0.23), имеем (см. доказательство теоремы 6 в части "необходимость")

$$
\begin{gathered}
\left\|f^{(r)}\right\| \leq C_{65}(r) E_{n}\left(f^{(r)}\right)+\left\|T_{n}^{(r)}(f)\right\| \leq C_{65}(r) C_{1}(k) \omega_{k}\left(f^{(r)} ; \frac{\pi}{n}\right)+\left\|T_{n}^{(r)}(f)\right\| \\
\leq C_{65}(r) C_{1}(k) C_{21}(k, r)\left\{n^{-k} \sum_{\nu=1}^{n} \nu^{k+r-1} E_{\nu-1}(f)+\sum_{\nu=n+1}^{\infty} \nu^{r-1} E_{\nu-1}(f)\right\}+C_{19}(r) \sum_{\nu=1}^{n} \nu^{r-1} E_{\nu-1}(f) \\
\leq C_{65}(r) C_{1}(k) C_{21}(k, r)\left\{n^{-k} n^{k} \sum_{\nu=1}^{n} \nu^{r-1} E_{\nu-1}(f)+\sum_{\nu=n+1}^{\infty} \nu^{r-1} E_{\nu-1}(f)\right\}+C_{19}(r) E(f ; r)
\end{gathered}
$$




$$
=C_{65}(r) C_{1}(k) C_{21}(k, r) E(f ; r)+C_{19}(r) E(f ; r)=\left(C_{65}(r) C_{1}(k) C_{21}(k, r)+C_{19}(r)\right) E(f ; r) .
$$

3 а м е ч а н и е 3 . Неравенство (0.21) можно также получить привлечением лишь неравенства (0.20) (справедливость которого обеспечивается выполнением неравенства $(0.22)$ ), применение которого в неравенстве (1.4) приводит к оценке:

$$
\omega_{k}\left(f^{(r)} ; \frac{\pi}{n}\right) \leq C_{14}(k) C_{20}(r)\left\{2 n^{-k} \sum_{\nu=1}^{n} \nu^{k+r-1} E_{\nu-1}(f)+\sum_{\nu=n+1}^{\infty} \nu^{r-1} E_{\nu-1}(f)\right\}, \quad n \in \mathbb{N} .
$$

Автор благодарен А.Г.Бабенко за внимательное прочтение текста работы и полезные замечания, которые привели к значительному улучшению изложения, а также признателен В. В. Шевченко за неоценимую техническую помощь при оформлении данной статьи.

\section{СПИСОК ЛИТЕРАТУРЫ}

1. Стечкин С.Б. О порядке наилучших приближений непрерывных функций // Изв. АН СССР. Сер. математическая. 1951. Т. 15, № 3. С. 219-242.

2. Стечкин С.Б. О порядке наилучших приближений непрерывных функций // Докл. АН СССР. 1949. T. 65, № 2. С. 135-137.

3. Стечкин С.Б. Обобщение некоторых неравенств С.Н.Бернштейна // Докл. АН СССР. 1948. T. 60, № 9. C. 1511-1514.

4. Jackson D. Über die Genauigkeit der Annäherung stetiger Funktionen durch ganze rationale Funktionen gegebenen Grades und trigonometrische Summen gegebener Ordnung. InauguralDissertation. Göttingen, 1911. S. 1-99.

5. Jackson D. On approximation by trigonometric sums and polynomials // Trans. Amer. Math. Soc. 1912. Vol. 13, no. 4. P. 491-515.

6. Jackson D. The theory of approximation. N.-Y., 1930. 178 p. (Amer. Math. Soc. Colloquium Publ.; vol. 11.)

7. Zygmund A. Smooth functions // Duke Math. J. 1945. Vol. 12, no. 1. P. 47-76. doi: 10.1215/S00127094-45-01206-3.

8. Vallée Poussin Ch.-J. Leçons sur l'approximation des fonctions d'une variable réelle. Paris: GauthierVillars, 1919. $150 \mathrm{p}$.

9. Ахиезер Н.И. Лекции по теории аппроксимации. 1-е изд. М.-Л.: ГИТТЛ, 1947. 324 с.; 2-е изд. М.: Наука, 1965. 408 с.

10. Тиман А.Ф. Теория приближения функций действительного переменного. М.: Физматгиз, 1960. $624 \mathrm{c}$.

11. Бернштейн С.Н. О свойствах однородных функциональных классов // Докл. АН СССР. 1947. T. 57, № 2. С. 111-114.

12. Гейт В.Э. Теоремы вложения для некоторых классов периодических непрерывных функций // Изв. вузов. Математика. 1972. № 4 (119). С. 67-77.

13. Жук В.В. Аппроксимация периодических функций. Л.: Изд-во Ленингр. ун-та, 1982. 368 с.

14. Натансон Г.И., Тиман М.Ф. Средние геометрические последовательности наилучших приближений // Вестн. Ленингр. ун-та. 1979. Сер. № 19, вып. 4. С. 50-52.

15. Натансон И.П. Конструктивная теория функций. М.-Л.: ГИТТЛ, 1949. 688 с.

16. Дзядык В.К. Введение в теорию равномерного приближения функций. М.: Наука, 1977. 512 с.

17. Бари Н.К. Тригонометрические ряды. М.: Физматгиз, 1961. 936 с.

18. Жук В.В. Об одном методе суммирования рядов Фурье. Ряды Фурье с положительными коэффициентами // Исследования по некоторым проблемам конструктивной теории функций: сб. науч. тр. Ленингр. механ. ин-та. № 50. Л., 1965. С. 73-92.

19. Белов А.С. О порядковых оценках наилучших приближений и модулей непрерывности суммы тригонометрического ряда с квазимонотонными коэффициентами // Мат. заметки. 1992. Т. 51, № 4. C. 132-134.

20. Зигмунд А. Тригонометрические ряды: в 2 т. М.: Мир, 1965. Т. 1.616 с.; Т. 2. 538 с.

21. Riesz M. Eine trigonometrische Interpolationsformel und einige Ungleichungen für Polynome // Jahresber. Deutsch. Math.-Verein. 1914. Bd. 23. S. 354-368. 
22. Никольский С.М. Обобщение одного неравенства С. Н. Бернштейна // Докл. АН СССР. 1948. T. LX, № 9. C. 1507-1510.

23. Тиман А.Ф., Тиман М.Ф. Обобщенный модуль непрерывности и наилучшее приближение в среднем // Докл. АН СССР. 1950. Т. 71, № 1. С. 17-20.

24. Бари Н.К., Стечкин С.Б. Наилучшие приближения и дифференциальные свойства двух сопряженных функций // Тр. Моск. мат. об-ва. 1956. Т. 5. С. 483-522.

25. Тиман А.Ф., Тиман М.Ф. О зависимости между модулями гладкости функций, заданных на всей вещественной оси // Докл. АН СССР. 1957. Т. 113, № 5. С. 995-997.

26. Salem R. Sur certaines fonctions continues et les propriétés de leurs séries de Fourier // Comptes Rendus de L’Acad. des Sci. 1935. Vol. 201, № 16. P. 703-705.

27. Salem R. Essais sur les sèries trigonometriques: Théses le grade de docteur ès-sciences mathématiques. Paris, $1940.85 \mathrm{p}$.

28. Бернштейн С.Н. О наилучшем приближении непрерывных функций посредством многочленов данной степени, I // Сообщения Харьков. мат. об-ва. Вторая сер. 1912. Т. 13, вып. 2-3. С. 49-144.

29. Quade E.S. Trigonometric approximation in the mean // Duke Math. J. 1937. Vol. 3, № 3. P. 529-543. doi: 10.1215/S0012-7094-37-00342-9.

Поступила 02.06.2020

После доработки 28.08.2020

Ильясов Ниязи Аладдин оглы

Принята к публикации 21.09.2020

канд. физ.-мат. наук, доцент

доцент кафедры математического анализа

Бакинский государственный университет

г. Баку;

Московский центр фундаментальной и прикладной математики

г. Москва

e-mail: niyazi.ilyasov@gmail.com

\section{REFERENCES}

1. Stechkin S.B. On the order of the best approximations of continuous functions. Izv. Akad. Nauk SSSR. Ser. Mat., 1951, vol. 15, no. 3, pp. 219-242 (in Russian).

2. Stechkin S.B. On the order of the best approximations of continuous functions. Dokl. Akad. Nauk SSSR, 1949 , vol. 65 , no. 2 , pp. 135-137 (in Russian).

3. Stechkin S.B. A generalization of some S. Bernstein's inequalities. Dokl. Akad. Nauk SSSR, 1948, vol. 60, no. 9, pp. 1511-1514 (in Russian).

4. Jackson D. Über die Genauigkeit der Annäherung stetiger Funktionen durch ganze rationale Funktionen gegebenen Grades und trigonometrische Summen gegebener Ordnung. Inaugural-Dissertation. Göttingen, 1911, $99 \mathrm{p}$.

5. Jackson D. On approximation by trigonometric sums and polynomials. Trans. Amer. Math. Soc., 1912, vol. 13 , no. 4, pp. 491-515. DOI: 10.2307/1988583 .

6. Jackson D. The theory of approximation. Ser. Amer. Math. Soc. Colloquium Publ., vol. 11. N.-Y.: AMS, 1930, 178 p. ISBN: 978-1-4704-3160-0.

7. Zygmund A. Smooth functions. Duke Math. J., 1945, vol. 12, no. 1, pp. 47-76. doi: 10.1215/S0012-7094-45-01206-3.

8. Vallée Poussin Ch.-J. Leçons sur l'approximation des fonctions d'une variable réelle. Paris: GauthierVillars, 1919, $150 \mathrm{p}$.

9. Achieser N.I. Theory of approximation, Reprint of the 1956, N Y: Dover Publ., 1992, 307 p. ISBN: 0486671291 . Original Russian text published in Akhiezer N.I. Lektsii po teorii approksimatsii. 1st ed.: Moscow; Leningrad: GITTL, 1947, 324 p.; 2nd ed.: Moscow: Nauka Publ., 1965, 408 p.

10. Timan A.F. Theory of approximation of functions of a real variable. Oxford; London; N.-Y.: Pergamon Press, 1963, 655 p. ISBN: 048667830X. Original Russian text published in Timan A.F. Teoriya priblizheniya funktsii deystvitel'nogo peremennogo. Moscow: Fizmatgiz Publ., 1960, 624 p.

11. Bernstein S.N. On properties of homogeneous functional classes. Dokl. AN SSSR, 1947, vol. 57, no. 2, pp. 111-114 (in Russian). 
12. Gheit V.E. Imbedding theorems for certain classes of continuous periodic functions. Izv. Vyssh. Uchebn. Zaved. Mat., 1972, no. 4 (119), pp. 67-77 (in Russian).

13. Zhuk V.V. Approksimatsiya periodicheskikh funktsii [Approximation of periodic functions]. Leningrad: Leningrad Univ. Publ., 1982, 368 p. (in Russian).

14. Natanson G.I., Timan M.F. Geometric means of a sequence of best approximations. Vestn. Leningr. Univ., 1979, ser. 19, no. 4, pp. 50-52 (in Russian).

15. Natanson I.P. Constructive function theory. Vol. I. Uniform approximation. N Y: Frederick Ungar Publishing Co., 1964, 232 p. ISBN: 9780094551503. Original Russian text published in Natanson I.P. Konstruktivnaya teoriya funktsii. Moscow; Leningrad: GITTL, 1949, 688 p.

16. Dzyadyk V.K. Vvedenie v teoriyu ravnomernogo priblizheniya funktsii polinomami [Introduction to the Theory of Uniform Approximation of Functions by Polynomials]. Moscow: Nauka, 1977, 512 p. (in Russian).

17. Bary N.K. A treatise on trigonometric series, vols. I,II. Oxford; N Y: Pergamon Press, 1964, vol. 1, 553 p.; vol. 2, 508 p. doi: 10.1002/zamm.19650450531. Original Russian text published in Bari N.K. Trigonometricheskie ryady. Moscow: Fiz.-Mat.Giz. Publ, 1961, 936 p.

18. Zhuk V.V. On the one method for summing Fourier series. Fourier series with positive coefficients. In: Studies on Some Problems of Constructive Function Theory, Collection of Scientific Works of the Leningrad Mechanical Institute, no. 50, 1965, pp. 73-92 (in Russian).

19. Belov A.S. Order estimates for best approximations and moduli of continuity of the sum of a trigonometric series with Quasi-monotone coefficients. Math. Notes, 1992, vol. 51, no. 4, pp. 417-419. doi: $10.1007 / \mathrm{BF} 01250558$.

20. Zygmund A. Trigonometric series. Vols. I \& II. Cambridge: Cambridge Univ. Press, 2003, 747 p. ISBN: 0-521-89053-5. Translated to Russian under the title Trigonometricheskie rjady. Moscow: Mir Publ., 1965, vol. I, 616 p.; vol. II, 538 p.

21. Riesz M. Eine trigonometrische Interpolationsformel und einige Ungleichungen für Polynome. Jahresber. Deutsch. Math.-Verein., 1914, bd. 23, s. 354-368. doi: 10.1007/978-3-642-37535-4_13 .

22. Nikol'skii S.M. A generalization of an inequality of S.N. Bernstein. Doklady Akad. Nauk SSSR, 1948, vol. 60, no. 9, pp. 1507-1510 (in Russian).

23. Timan A.F., Timan M.F. Generalized modulus of continuity and best approximation in the mean. Dokl. Akad. Nauk SSSR, 1950, vol. 71, no. 1, pp. 17-20 (in Russian).

24. Bari N.K., Stechkin S.B. Best approximations and differential properties of two conjugate functions. Trudy Mosk. Mat. Obsh., 1956, vol. 5, pp. 483-522 (in Russian).

25. Timan A.F., Timan M.F. On the relation between the smoothness moduli of functions given all along the axis of reals. Dokl. Akad. Nauk SSSR, 1957, vol. 113, no. 5, pp. 995-997 (in Russian).

26. Salem R. Sur certaines fonctions continues et les propriétés de leurs séries de Fourier. Comptes Rendus de L'Acad. des Sci., 1935, vol. 201, no. 16, pp. 703-705.

27. Salem R. Essais sur les sèries trigonometriques. Théses le grade de docteur ès-sciences mathématiques. Paris, 1940, 85 p.

28. Bernstein S.N. Best approximation of continuous functions by polynomials of a given degree, I. Soobshcheniya Har'kov. mat. ob-va. Vtoraya ser., 1912, vol. 13, no. 2-3, pp. 49-144 (in Russian).

29. Quade E.S. Trigonometric approximation in the mean. Duke Math. J., 1937, vol. 3, no. 3, pp. $529-543$. doi: 10.1215/S0012-7094-37-00342-9 .

Received June 2, 2020

Revised August 28, 2020

Accepted September 21, 2020

Niyazi Aladdin ogly Il'yasov, Cand. Sci. (Phys.-Math.), Baku State University, Baku, Azerbaijan; Moscow Center for Fundamental and Applied Mathematics, Moscow, Russia, e-mail: niyazi.ilyasov@gmail.com .

Cite this article as: N. A. Il'yasov. Some supplements to S. B. Stechkin's inequalities in direct and inverse theorems on the approximation of continuous periodic functions, Trudy Instituta Matematiki i Mekhaniki UrO RAN, 2020, vol. 26, no. 4, pp. 155-181. 